\title{
SOME PROBLEMS IN THE CALCULUS OF VARIATIONS
}

\author{
Arrigo Cellina
}

Annual Lecture dedicated to the memory of Professor Andrzej Lasota

\begin{abstract}
We present some results and open problems in the Calculus of Variations.
\end{abstract}

\section{Introduction}

The text that follows is a very expanded version of the Andrzej Lasota Commemorative Lecture, given in Katowice in January 2016. The Lecture was presented to an audience of mathematicians, not necessarily specialists in the Calculus of Variations. This text, although it is a survey of several recent results and open problems in the Calculus of Variations that have been of great interest to the author, has retained the character of being addressed to non-specialists. The topics that are presented are: the necessary conditions; the existence of solutions to problems of slow growth and some problems concerning the regularity of solutions. Very little or none preliminary knowledge of these topics is required.

Received: 03.01.2017. Accepted: 28.03.2017. Published online: 30.06.2017.

(2010) Mathematics Subject Classification: 35B65, 35D30, 35J61.

Key words and phrases: regularity of solutions, Lagrangians, Euler-Lagrange equation. 


\section{Necessary conditions and the validity of the Euler-Lagrange equation}

The purpose of this section is to investigate the necessary conditions satisfied by a solution. Hence, we assume that a solution to a given variational problem exists and wish to find some properties of it, that hopefully will single out this solution from the other competing functions satisfying the same boundary conditions. We will not seek to prove results in the most general conditions; rather, we shall consider essentially problems depending only on the variable $\nabla v$ and we will discuss the various difficulties arising, under different assumptions, when we try to establish the validity of necessary conditions.

\section{The classical approach and a discussion of the problem}

Let $L$ be a convex and differentiable function and consider the problem of minimizing

$$
\int_{\Omega} L(\nabla v(x)) d x
$$

under suitable boundary conditions. We assume that we have a solution $u$ and would like to find a condition satisfied by the solution. In a sense, this condition will make the solution special among all the other functions satisfying the same boundary conditions.

Take a variation $\eta$, a very regular function that vanishes outside some compact subset of $\Omega$, say $\eta \in C_{0}^{\infty}(\Omega)$. This choice implies that, for every $\varepsilon$, the function $u+\varepsilon \eta$ satisfies the same boundary conditions as $u$ does, and, being $\eta$ smooth, there is hope that $\int_{\Omega} L(\nabla(u+\varepsilon \eta)(x)) d x$ be finite. Since $u$ is a solution, we must have $\int_{\Omega} L(\nabla(u+\varepsilon \eta)) d x \geq \int_{\Omega} L(\nabla u) d x$ and, taking $\varepsilon>0$,

$$
\int_{\Omega} \frac{1}{\varepsilon}[L(\nabla(u+\varepsilon \eta))-L(\nabla u)] d x \geq 0 .
$$

From the convexity and differentiability of $L$ we infer that $\frac{1}{\varepsilon}[L(\nabla)+$ $\varepsilon \eta))-L(\nabla u)]$ is monotonically increasing with $\varepsilon$ so that, as $\varepsilon$ decreases to 0 , the integrand decreases (pointwise) to $\langle\nabla L(\nabla u(x)), \nabla \eta(x)\rangle$. Hence, whenever we can pass to the limit under the integral sign, we obtain from 2.2 that

$$
\int_{\Omega}\langle\nabla L(\nabla u(x)), \nabla \eta(x)\rangle d x \geq 0 .
$$


If the above inequality has been proved for every $\eta \in C_{0}^{\infty}(\Omega)$, then, by taking $-\eta$ instead of $\eta$, we finally have: for every $\eta \in C_{0}^{\infty}(\Omega)$, we must have

$$
\int_{\Omega}\langle\nabla L(\nabla u(x)), \nabla \eta(x)\rangle d x=0 .
$$

This is the Euler-Lagrange equation (for problem (2.1)). It is a very remarkable equation, that has strong implications for the properties of the solution itself, in particular for its regularity.

The purpose of the present section is to present some conditions for its validity.

The first tool that comes to mind, when we have pointwise convergence of the integrands, is the Dominated Convergence Theorem; we will discuss here when this approach is possible.

Let $L$ be a convex and differentiable function; fix $v$ and $w$. We begin by noticing that the convergence $\frac{L(v+\varepsilon w)-L(v)}{\varepsilon} \rightarrow\langle\nabla L(v), w\rangle$ implies that, for $\varepsilon>0$, both

$$
\left(\frac{L(v+\varepsilon w)-L(v)}{\varepsilon}\right)^{+} \rightarrow\langle\nabla L(v), w\rangle^{+}
$$

and

$$
\left(\frac{L(v+\varepsilon w)-L(v)}{\varepsilon}\right)^{-} \rightarrow\langle\nabla L(v), w\rangle^{-}
$$

Moreover, as it is well known, from the convexity of $L$ we infer that the map

$$
\varepsilon \rightarrow \frac{L(v+\varepsilon w)-L(v)}{\varepsilon}
$$

is monotonic non-decreasing.

Hence, given a decreasing sequence $\left(\varepsilon_{n}\right)$, with $\varepsilon_{n} \geq 0$ and $\varepsilon_{n} \rightarrow 0$, consider the quotient $\frac{L\left(\nabla u(x)+\varepsilon_{n} \nabla \eta(x)\right)-L(\nabla u(x))}{\varepsilon_{n}}$ : for $x$ such that $\langle\nabla L(\nabla u(x), \nabla \eta(x)\rangle \geq$ 0 , we have $L\left(\nabla u(x)+\varepsilon_{n} \nabla \eta(x)\right)-L(\nabla u(x)) \geq\langle\nabla L(\nabla u(x)), \varepsilon \nabla \eta(x)\rangle \geq 0$ and hence

$$
\begin{aligned}
\left|\frac{L\left(\nabla u(x)+\varepsilon_{n} \nabla \eta(x)\right)-L(\nabla u(x))}{\varepsilon_{n}}\right| & =\frac{L\left(\nabla u(x)+\varepsilon_{n} \nabla \eta(x)\right)-L(\nabla u(x))}{\varepsilon_{n}} \\
& \leq \frac{L\left(\nabla u(x)+\varepsilon_{1} \nabla \eta(x)\right)-L(\nabla u(x))}{\varepsilon_{1}}
\end{aligned}
$$


and, in order to have an integrable function that dominates the sequence of quotients on the set $\{x:\langle\nabla L(\nabla u(x)), \nabla \eta(x)\rangle \geq 0\}$, we have only to make sure that the map $x \rightarrow L\left(\nabla u(x)+\varepsilon_{1} \nabla \eta(x)\right)$ is integrable on that set.

Next, consider $x$ such that $\langle\nabla L(\nabla u(x)), \nabla \eta(x)\rangle<0$ : when $n$ is sufficiently large,

$$
\begin{gathered}
\left|\frac{L\left(\nabla u(x)+\varepsilon_{n} \nabla \eta(x)\right)-L(\nabla u(x))}{\varepsilon_{n}}\right| \\
=-\frac{L\left(\nabla u(x)+\varepsilon_{n} \nabla \eta(x)\right)-L(\nabla u(x))}{\varepsilon_{n}} \\
\quad \leq-\langle\nabla L(\nabla u(x)), \nabla \eta(x)\rangle=|\langle\nabla L(\nabla u(x)), \nabla \eta(x)\rangle|
\end{gathered}
$$

and the sequence $\left|\frac{L\left(\nabla u(x)+\varepsilon_{n} \nabla \eta(x)\right)-L(\nabla u(x))}{\varepsilon_{n}}\right|$ has $|\langle\nabla L(\nabla u(x)), \nabla \eta(x)\rangle|$ as a dominant.

Hence, to apply the Dominated Convergence Theorem to the proof of the validity of the Euler-Lagrange equation, we must know a priori that both $L\left(\nabla u(x)+\varepsilon_{1} \nabla \eta(x)\right)$ and $|\langle\nabla L(\nabla u(x)), \nabla \eta(x)\rangle|$ are integrable.

However, notice that, in general, from the information that

$$
\int_{\Omega} L(\nabla v(x)) d x<\infty
$$

(this is the information that we do have) we cannot infer that

$$
\int_{\Omega}|\nabla L(\nabla v(x))| d x<\infty
$$

Consider the following simple example.

Let $\Omega$ be the one-dimensional interval $I=[0, b]$, let $L(\xi)=e^{|\xi|^{2}}$ and set

$$
v^{\prime}(x)=\sqrt{-\ln \left(x|\ln (x)|^{\frac{4}{3}}\right)} .
$$

We have $\int_{I} e^{\left|v^{\prime}(x)\right|^{2}} d x=\int_{I} \frac{1}{x|\ln (x)|^{\frac{4}{3}}} d x<\infty$ while

$$
\int_{I}\left|L^{\prime}\left(v^{\prime}(x)\right)\right| d x=\int_{I} 2 \sqrt{-\ln \left(x|\ln (x)|^{\frac{4}{3}}\right)} \frac{1}{x|\ln (x)|^{\frac{4}{3}}} d x .
$$

We claim: for sufficiently small $b, 0<x \leq b$ implies that

$$
-\ln \left(x|\ln (x)|^{\frac{4}{3}}\right) \geq \frac{1}{2}|\ln (x)|^{\frac{4}{3}}
$$


or, equivalently, that $\frac{1}{x\left(\ln \left(\frac{1}{x}\right)\right)^{\frac{4}{3}}} \geq \frac{1}{x^{\frac{1}{2}}}$. But this last statement is true since we have $\lim _{x \rightarrow 0^{+}} x^{\frac{1}{2}}\left(\ln \left(\frac{1}{x}\right)\right)^{\frac{4}{3}}=0$, so that, for $x$ small, $x^{\frac{1}{2}}\left(\ln \left(\frac{1}{x}\right)\right)^{\frac{4}{3}}<1$. Then, by this claim,

$$
\int_{I}\left|L^{\prime}\left(v^{\prime}(x)\right)\right| d x \geq \int_{I} \sqrt{2}|\ln (x)|^{\frac{2}{3}} \frac{1}{x|\ln (x)|^{\frac{4}{3}}} d x=\int_{I} \sqrt{2} \frac{1}{x|\ln (x)|^{\frac{2}{3}}} d x=+\infty .
$$

Hence, $L^{\prime}\left(v^{\prime}\right) \notin \mathrm{L}^{1}$ even though $L\left(v^{\prime}\right) \in \mathrm{L}^{1}$.

\section{The validity of the Euler-Lagrange equation via dominated convergence}

For a function $L(x, u, \xi)$ we shall denote $\partial L_{\xi}$ and $\nabla_{\xi} L$ the subdifferential and the gradient of $L$ with respect to its last variable. Let us begin by the following lemma on the growth of the gradient (more generally, of the subgradient) of a convex function.

Lemma 2.1. Let $L(x, u, \xi)$ be such that, for every $x, u$, the map $\xi \rightarrow$ $L(x, u, \xi)$ is convex and assume that there exist constants $p \geq 1$ and $C$ such that $L(x, u, \xi) \leq C\left(1+|\xi|^{p}\right)$. Then, there exists a constant $K$ such that, for every $x, u, \xi$ and every $d \in \partial L_{\xi}(x, u, \xi)$,

$$
|d| \leq K\left(1+|\xi|^{p-1}\right) .
$$

In particular, when $L$ is differentiable, we have $\left|\nabla_{\xi} L(x, u, \xi)\right| \leq K\left(1+|\xi|^{p-1}\right)$.

Proof. We first notice that, by convexity, $(a+b)^{p} \leq \frac{1}{2}(2 a)^{p}+\frac{1}{2}(2 b)^{p}=$ $2^{p-1}\left(a^{p}+b^{p}\right)$.

Given $x, u, \xi$ and $\zeta$, we have $L(x, u, \xi+\zeta) \geq L(x, u, \xi)+\langle d, \zeta\rangle$, with $d$ any element of the subdifferential $\partial_{\xi} L(x, u, \xi)$, and hence,

$$
\begin{aligned}
\langle d, \zeta\rangle & \leq|L(x, u, \xi+\zeta)|+|L(x, u, \xi)| \\
& \leq C\left(1+|\xi+\zeta|^{p}\right)+C\left(1+|\xi|^{p}\right) \\
& \leq C\left(1+(|\xi|+|\zeta|)^{p}\right)+C\left(1+|\xi|^{p}\right) \leq \tilde{C}\left(1+|\xi|^{p}+|\zeta|^{p}\right) .
\end{aligned}
$$

Choose $\zeta=v\left(1+|\xi|^{p}\right)^{\frac{1}{p}}$ with $|v|=1$, so that $|\zeta|^{p}=1+|\xi|^{p}$, to obtain $\langle d, \zeta\rangle \leq 2 \tilde{C}\left(1+|\xi|^{p}\right)$ and $\langle d, v\rangle \leq 2 \tilde{C}\left(1+|\xi|^{p}\right)^{1-\frac{1}{p}}$. For each $t \geq 0$, we have 
$\left(1+t^{p}\right)^{1-\frac{1}{p}} \leq\left(1+t^{p-1}\right) ;$ in fact, both take the value 1 at 0 and

$$
\frac{d}{d t}\left(1+t^{p}\right)^{1-\frac{1}{p}}=(p-1) \frac{t^{p-1}}{\left(1+t^{p}\right)^{\frac{1}{p}}} \leq(p-1) \frac{t^{p-1}}{t}=\frac{d}{d t}\left(1+t^{p-1}\right) .
$$

Hence,

$$
\langle d, v\rangle \leq K\left(1+|\xi|^{p-1}\right)
$$

Finally, take $v=\frac{d}{|d|}$ when $|d|>0$ to obtain $|d| \leq K\left(1+|\xi|^{p-1}\right)$. When $d=0$, the inequality is obviously true.

For the case where $L$ depends only on the variables $x$ and $\xi$, we have the following simple result on the validity of the Euler-Lagrange equation.

THEOREM 2.2. Let $L(x, \xi)$ satisfy Carathéodory conditions and be such that, for every $x$, the map $\xi \rightarrow L(x, \xi)$ is convex and differentiable. Moreover, assume that there exist constants $p \geq 1$ and $C$ such that $L(x, \xi) \leq C\left(1+|\xi|^{p}\right)$. Let $u^{0} \in W^{1, p}(\Omega)$ and let $u$ be a solution to the problem of minimizing

$$
\int_{\Omega} L(x, \nabla u(x)) d x
$$

on $u^{0}+W_{0}^{1, p}(\Omega)$. Then, for every $w \in W_{0}^{1, p}(\Omega)$, we have

$$
\int_{\Omega}\left\langle\nabla_{\xi} L(x, \nabla u(x)), \nabla w(x)\right\rangle d x=0 .
$$

ProOF. By definition of solution, for $\varepsilon>0$,

$$
0 \leq \frac{1}{\varepsilon} \int_{\Omega}[L(x, \nabla u(x)+\varepsilon \nabla w(x))-L(x, \nabla u(x))] d x
$$$$
=\frac{1}{\varepsilon} \int_{\left\{x \in \Omega:\left\langle\nabla_{\xi} L(x, \nabla u(x)), \nabla w(x)\right\rangle \geq 0\right\}}[L(x, \nabla u(x)+\varepsilon \nabla w(x))-L(x, \nabla u(x))] d x
$$

$+\frac{1}{\varepsilon} \int_{\left\{x \in \Omega:\left\langle\nabla_{\xi} L(x, \nabla u(x)), \nabla w(x)\right\rangle<0\right\}}[L(x, \nabla u(x)+\varepsilon \nabla w(x))-L(x, \nabla u(x))] d x$.

Since $L(x, \nabla u(x)+\nabla w(x)) \leq \frac{1}{2} L(x, 2 \nabla u(x))+\frac{1}{2} L(x, 2 \nabla w(x)) \leq \frac{1}{2} 2^{p} C[(1+$ $\left.|\nabla u(x)|^{p}\right)+\left(1+|\nabla w(x)|^{p}\right)$, the assumption that $w \in W^{1, p}(\Omega)$ assures that $L(\cdot, \nabla u+\nabla w) \in \mathrm{L}^{1}(\Omega)$. Hence, on the set

$$
\left\{x \in \Omega:\left\langle\nabla_{\xi} L(x, \nabla u(x)), \nabla w(x)\right\rangle \geq 0\right\},
$$


each integrand $\frac{1}{\varepsilon}[L(\cdot, \nabla u(\cdot)+\varepsilon \nabla w(\cdot))-L(\cdot, \nabla u(\cdot))]$ is dominated by the integrable function

$$
L(\cdot, \nabla u(\cdot)+\nabla w(\cdot))-L(\cdot, \nabla u(\cdot)) .
$$

On the set $\left\{x \in \Omega:\left\langle\nabla_{\xi} L(x, \nabla u(x)), \nabla w(x)\right\rangle<0\right\}$, by $(2.3), \frac{1}{\varepsilon} \mid L(\cdot, \nabla u(\cdot)+$ $\varepsilon \nabla w(\cdot))-L(\cdot, \nabla u(\cdot)) \mid$ is dominated by $\left|\left\langle\nabla_{\xi} L(\cdot, \nabla u(\cdot)), \nabla w(\cdot)\right\rangle\right|$ and this last function is integrable by Lemma 2.1 and the assumption that $w \in W^{1, p}(\Omega)$. In fact

$$
\begin{aligned}
\int_{\Omega}\left|\left\langle\nabla_{\xi} L(\cdot, \nabla u(\cdot)), \nabla w(\cdot)\right\rangle\right| & \leq \int_{\Omega} K\left(1+|\nabla u|^{p-1}\right)|\nabla w| \\
& \leq K\|\nabla w\|_{L^{1}}+K\left\||\nabla u|^{p-1}\right\|_{L^{q}}\|\nabla w\|_{L^{p}} \\
& =K\|\nabla w\|_{L^{1}}+K\left(\|\nabla u\|_{L^{p}}\right)^{\frac{p}{q}}\|\nabla w\|_{L^{p}}
\end{aligned}
$$

Since, pointwise,

$$
\frac{1}{\varepsilon}[L(x, \nabla u(x)+\varepsilon \nabla w(x))-L(x, \nabla u(x))] \rightarrow\left\langle\nabla_{\xi} L(x, \nabla u(x)), \nabla w(x)\right\rangle,
$$

by dominated convergence we obtain from (2.4) that

$$
0 \leq \int_{\Omega}\left\langle\nabla_{\xi} L(x, \nabla u(x)), \nabla w(x)\right\rangle d x .
$$

Taking $-w$ instead of $w$, the result is proved.

Notice that Theorem 2.2 requires the a priori knowledge that the solution $u$ be in $W^{1, p}(\Omega)$, and this fact, in general, can be assured only by requiring some additional growth assumption on $L$ : for instance, assuming that there exist a function $\alpha \in \mathrm{L}^{1}(\Omega)$ and a positive constant $\beta$ such that

$$
L(x, \xi) \geq \alpha(x)+\beta|\xi|^{p} .
$$

\section{Stretching the dominated convergence approach}

For convex Lagrangians $L$ growing, in the variable $\xi$, like $|\xi|^{p}$, the gradient of $L$ grows like $|\xi|^{p-1}$, and this fact is exploited in Theorem 2.2 to allow the variation $w$ to be in $W_{0}^{1, p}(\Omega)$. When we reach exponential growth, this is not true anymore; however, the method based on dominated convergence can 
be used also in this limiting case, restricting the class of allowed variations to $\eta \in C_{c}^{1}(\Omega)$. In the case of exponential growth, the connection between the growth of $L$ and the growth of $\nabla L$, that is expressed by Lemma 2.1, is replaced by the condition appearing in the following definition.

Exponential growth condition. For every open $O \subset \subset \Omega$ there exists a constant $c$ such that, for almost every $x \in O,|\xi| \geq 1$ implies $\left|\nabla_{\xi} L(x, \xi)\right| \leq$ $c L(x, \xi)$.

Maps like $e^{\sqrt{1+|\xi|^{2}}}$ or $\frac{e^{|\xi|}+e^{-|\xi|}}{2}$ satisfy this assumption.

Theorem 2.3. Let $L(x, \xi)$ satisfy Carathéodory conditions and be such that, for every $x$, the map $\xi \rightarrow L(x, \xi)$ is convex and differentiable. Moreover, assume that the exponential growth condition is satisfied. Let $u^{0} \in W^{1,1}(\Omega)$ be such that $\int_{\Omega} L\left(x, \nabla u^{0}(x)\right) d x<\infty$ and let $u$ be a solution to the problem of minimizing

$$
\int_{\Omega} L(x, \nabla u(x)) d x
$$

on $u^{0}+W_{0}^{1,1}(\Omega)$. Then, for every $\eta \in C_{c}^{1}(\Omega)$, we have

$$
\int_{\Omega}\left\langle\nabla_{\xi} L(x, \nabla u(x)), \nabla \eta(x)\right\rangle d x=0 .
$$

Proof. Fix $\eta$, set $O=\operatorname{supp}(\eta)$ and set $D=\sup \{|\nabla \eta|\}$. To apply the Dominated Convergence Theorem as in Theorem 2.2, it is sufficient to show that $L(\cdot, \nabla u(\cdot)+\nabla \eta(\cdot))$ is integrable on the set

$$
\left\{x \in \Omega:\left\langle\nabla_{\xi} L(x, \nabla u(x)), \nabla w(x)\right\rangle \geq 0\right\}
$$

and that $\left|\left\langle\nabla_{\xi} L(\cdot, \nabla u(\cdot)), \nabla \eta(\cdot)\right\rangle\right|$ is integrable on $\Omega$. This last condition follows directly from the definition. Since $\frac{d}{d t} L(x, a+t b)=\left\langle\nabla_{\xi} L(x, a+t b), b\right\rangle \leq$ $c|b| L(x, a+t b)$ we have

$$
L(x, a+b) \leq L(x, a) e^{c|b|}
$$

and hence

$$
L(x, \nabla u(x)+\nabla \eta(x)) \leq L(x, \nabla u(x)) e^{c D} .
$$

Both integrability conditions are satisfied; passing to the limit we obtain that

$$
\int_{\Omega}\left\langle\nabla_{\xi} L(x, \nabla u(x)), \nabla \eta(x)\right\rangle d x=0 .
$$




\section{Lagrangians of fast growth}

In this section we shall consider minimization problems without restriction on the growth of $L$ with respect to $|\xi|$, aiming at deriving the Euler-Lagrange equation. We cannot base the proof on dominated convergence, hence we should expect that the proof will be longer and less direct.

Consider again the variational problem

$$
\text { minimize } \int_{\Omega} L(x, \nabla v(x)) d x \text { on } u^{0}+W_{0}^{1,1}(\Omega) .
$$

Next lemma requires the convexity of $L$ but no growth assumption whatsoever. Let $u$ be the solution to the minimization problem, so that, in particular, $L(\cdot, \nabla u(\cdot)) \in \mathrm{L}^{1}(\Omega)$; let $v$ be such that also $L(\cdot, \nabla v(\cdot)) \in \mathrm{L}^{1}(\Omega)$; then, the result is stated in terms of $v-u$. Setting $\eta=v-u$, the integrability requirement on $L(\cdot, \nabla v(\cdot))$ amounts to asking that $L(\cdot, \nabla(u+\eta)(\cdot))$ be in $L^{1}(\Omega)$. For Lagrangians $L$ growing faster than exponential, even assuming that $\eta$ is very regular $\left(C^{\infty}\right)$ will not guarantee this integrability. Consider $L(\xi)=e^{|\xi|^{2}}$; then,

$$
e^{|\nabla u+\nabla \eta|^{2}}=e^{|\nabla u|^{2}+2\langle\nabla u, \nabla \eta\rangle+|\nabla \eta|^{2}}=e^{|\nabla \eta|^{2}} e^{2\langle\nabla u, \nabla \eta\rangle} e^{|\nabla u|^{2}}
$$

and, in principle, the map at the right hand side grows, in $\nabla u$, faster than $e^{|\nabla u|^{2}}$; hence, the integrability of $L(\cdot, \nabla(u+\eta)(\cdot))$ is not guaranteed.

Lemma 2.4. Let $\Omega$ be an open subset of $\mathbb{R}^{N}$, let $L \geq 0$ satisfy Carathéodory conditions; in addition, let $\xi \rightarrow L(x, \xi)$ be convex and differentiable and let $\nabla_{\xi} L$ also satisfy Carathéodory conditions. Let $u \in W_{\text {loc }}^{1,1}(\Omega)$ be a solution to problem 2.5 and let $v \in u+W_{0}^{1,1}(\Omega)$ be such that $L(\cdot, \nabla v(\cdot)) \in \mathrm{L}^{1}(\Omega)$. Then:

(i) $\left\langle\nabla_{\xi} L(\cdot, \nabla u), \nabla(v-u)\right\rangle \in \mathrm{L}^{1}(\Omega)$,

(ii) $\int_{\Omega}\left\langle\nabla_{\xi} L(\cdot, \nabla u), \nabla(v-u)\right\rangle d x \geq 0$.

Proof. Since $L$ is convex with respect to the variable $\xi$, it follows that for every $t \in[0,1]$

$$
L(x, \nabla u+t(\nabla v-\nabla u)) \leq L(x, \nabla u)+t[L(x, \nabla v)-L(x, \nabla u)]
$$

so that $L(\cdot, \nabla u+t(\nabla v-\nabla u)) \in \mathrm{L}^{1}(\Omega)$. Rewrite inequality 2.6 as

$$
\frac{1}{t}[L(x, \nabla u+t(\nabla v-\nabla u))-L(x, \nabla u)] \leq L(x, \nabla v)-L(x, \nabla u)
$$


that implies

$$
\frac{1}{t}(L(x, \nabla u+t(\nabla v-\nabla u))-L(x, \nabla u))^{+} \leq(L(x, \nabla v)-L(x, \nabla u))^{+} .
$$

The integrability of $L(x, \nabla v)-L(x, \nabla u)$ implies the integrability of $(L(x, \nabla v)-$ $L(x, \nabla u))^{+}$; we know that $\frac{1}{t}(L(x, \nabla u(x)+t(\nabla v(x)-\nabla u(x)))-L(x, \nabla u(x)))^{+}$ converges pointwise to $(\langle\nabla L(x, \nabla u(x)), \nabla(v-u)(x)\rangle)^{+}$and is dominated by the integrable function $(L(\cdot, \nabla v)-L(\cdot, \nabla u))^{+}$, so that

$$
\begin{aligned}
& \int_{\Omega}(\langle\nabla L(x, \nabla u(x)), \nabla(v-u)(x)\rangle)^{+} d x \\
= & \lim _{t_{n} \rightarrow 0^{+}} \int_{\Omega} \frac{1}{t_{n}}\left(L\left(x, \nabla u(x)+t_{n}(\nabla v(x)-\nabla u(x))\right)-L(x, \nabla u(x))\right)^{+} d x .
\end{aligned}
$$

On the other hand, apply Fatou's Lemma to the sequence of non-negative functions $\frac{1}{t_{n}}\left(L\left(x, \nabla u(x)+t_{n}(\nabla v(x)-\nabla u(x))\right)-L(x, \nabla u(x))\right)^{-}$to obtain

$$
\begin{aligned}
\int_{\Omega} & (\langle\nabla L(x, \nabla u(x)), \nabla(v-u)(x)\rangle)^{-} d x \\
& =\int_{\Omega} \lim _{t_{n} \rightarrow 0^{+}} \frac{1}{t_{n}}\left(L\left(x, \nabla u(x)+t_{n}(\nabla v(x)-\nabla u(x))\right)-L(x, \nabla u(x))\right)^{-} d x \\
& \leq \varliminf_{t_{n} \rightarrow 0^{+}} \int_{\Omega} \frac{1}{t_{n}}\left(L\left(x, \nabla u(x)+t_{n}(\nabla v(x)-\nabla u(x))\right)-L(x, \nabla u(x))\right)^{-} d x
\end{aligned}
$$

so that

$$
\begin{aligned}
& \int_{\Omega}-(\langle\nabla L(x, \nabla u(x)), \nabla(v-u)(x)\rangle)^{-} d x \\
& \quad \geq \varlimsup_{t_{n} \rightarrow 0^{+}} \int_{\Omega}-\frac{1}{t_{n}}\left(L\left(x, \nabla u(x)+t_{n}(\nabla v(x)-\nabla u(x))\right)-L(x, \nabla u(x))\right)^{-} d x
\end{aligned}
$$

and, combining (2.9) and the above inequality, we obtain

$$
\begin{aligned}
& \int_{\Omega}\langle\nabla L(x, \nabla u(x)), \nabla(v-u)(x)\rangle d x \\
& \quad \geq \lim _{t_{n} \rightarrow 0^{+}} \int_{\Omega} \frac{1}{t_{n}}\left(L\left(x, \nabla u(x)+t_{n}(\nabla v(x)-\nabla u(x))\right)-L(x, \nabla u(x))\right)^{+} d x
\end{aligned}
$$




$$
\begin{aligned}
& +\varlimsup_{t_{n} \rightarrow 0^{+}} \int_{\Omega}-\frac{1}{t_{n}}\left(L\left(x, \nabla u(x)+t_{n}(\nabla v(x)-\nabla u(x))\right)-L(x, \nabla u(x))\right)^{-} d x \\
& \quad=\varlimsup_{t_{n} \rightarrow 0^{+}} \int_{\Omega} \frac{1}{t_{n}}\left[L\left(x, \nabla u(x)+t_{n}(\nabla v(x)-\nabla u(x))\right)-L(x, \nabla u(x))\right] d x .
\end{aligned}
$$

Since $u$ is a solution, $\int_{\Omega}\left[L\left(x, \nabla u(x)+t_{n}(\nabla v(x)-\nabla u(x))\right)-L(x, \nabla u(x))\right] d x \geq$ 0 , thus proving assertion (ii) of the lemma.

We have shown that

$$
\int_{\Omega}\left(\left\langle\nabla_{\xi} L(x, \nabla u), \nabla(v-u)\right\rangle\right)^{-} \leq \int_{\Omega}\left(\left\langle\nabla_{\xi} L(x, \nabla u), \nabla(v-u)\right\rangle\right)^{+} .
$$

From 2.7,

$$
\frac{1}{t}[L(x, \nabla u+t(\nabla v-\nabla u))-L(x, \nabla u)]^{+} \leq(L(x, \nabla v(x))-L(x, \nabla u(x)))^{+}
$$

and we obtain

$$
\left\langle\nabla_{\xi} L(x, \nabla u(x)), \nabla(v-u)(x)\right\rangle^{+} \leq[L(x, \nabla v(x))-L(x, \nabla u(x))]^{+}
$$

hence $\left(\left\langle\nabla_{\xi} L(x, \nabla u(x)), \nabla(v-u)(x)\right\rangle\right)^{+} \in \mathrm{L}^{1}(\Omega)$, which - combined with 2.10 - gives assertion (i):

$$
\left\langle\nabla_{\xi} L(x, \nabla u(x)), \nabla(v-u)(x)\right\rangle \in \mathrm{L}^{1}(\Omega)
$$

and the proof is complete.

Next lemma requires the local boundedness of the solution $u$; alternatively, we can consider the following assumption, that would imply this local boundedness.

Assumption: For every $M$ there exists an integrable function $\alpha_{M}$ such that, for $x$ in $B(0, M) \cap \Omega$,

$$
L(x, \xi) \geq \alpha_{M}(x)+C|\xi|^{p}
$$

for some $p>N$.

LEMmA 2.5. Let $L: \Omega \times \mathbb{R}^{M}$ be non-negative, satisfying Carathéodory conditions and such that $\xi \rightarrow L(x, \xi)$ is convex. Moreover, assume that, for every positive $H$, there exists a function $\alpha_{H} \in \mathrm{L}^{1}(\Omega)$ such that $|\xi| \leq H$ implies $L(x, \xi) \leq \alpha_{H}(x)$. Let the solution $u$ to the problem of minimizing (2.5) be in $\mathrm{L}_{\mathrm{loc}}^{\infty}(\Omega)$. Then, $\left\langle\nabla_{\xi} L(\cdot, \nabla u(\cdot)), \nabla u(\cdot)\right\rangle \in \mathrm{L}_{\mathrm{loc}}^{1}(\Omega)$ and $\left|\nabla_{\xi} L(\cdot, \nabla u(\cdot))\right| \in$ $\mathrm{L}_{\text {loc }}^{1}(\Omega)$. 
Proof. Fix arbitrarily $x^{0} \in \Omega$, let $\delta$ be such that $B\left(x^{0}, 2 \delta\right) \subset \Omega$. Let $\vartheta \in C_{c}^{\infty}\left(B\left(x^{0}, 2 \delta\right)\right)$ be such that $\vartheta=1$ on $B\left(x^{0}, \frac{\delta}{2}\right)$ and $0 \leq \vartheta \leq 1$ elsewhere.

a) Since $u \in \mathrm{L}_{\text {loc }}^{\infty}(\Omega)$, choose $R$ so that $|u|<R$ on $B\left(x^{0}, 2 \delta\right)$ and consider the following variation:

$$
v(x)=\left\{\begin{array}{lll}
\max \{R(2 \vartheta(x)-1), u(x)\} & \text { if } \quad x \in B\left(x^{0}, 2 \delta\right), \\
u(x) & \text { if } \quad x \in \Omega \backslash B\left(x^{0}, 2 \delta\right) .
\end{array}\right.
$$

We have $v \in u+W_{0}^{1,1}(\Omega)$. Moreover,

$$
\nabla v(x)= \begin{cases}\nabla u(x) & \text { if } x \in \mathbb{C}(\operatorname{supp}(\vartheta)) \text { or if } x \in \operatorname{supp}(\vartheta) \\ & \text { but } u(x) \geq R(2 \vartheta(x)-1) \\ \nabla \vartheta(x) & \text { otherwise. }\end{cases}
$$

In particular, setting $H=\sup \{|\nabla \eta|\}$, we have $L(x, \nabla \eta(x)) \leq \alpha_{H}(x)$ and we obtain $L(\cdot, \nabla v(\cdot)) \in \mathrm{L}^{1}(\Omega)$. Now apply Lemma 2.4 to $v$ to infer that $\left\langle\nabla_{\xi} L(\cdot, \nabla u(\cdot)), \nabla(v-u)(\cdot)\right\rangle \in \mathrm{L}^{1}(\Omega)$, so that, in particular,

$$
\left\langle\nabla_{\xi} L(\cdot, \nabla u(\cdot)), \nabla(v-u)(\cdot)\right\rangle \in \mathrm{L}^{1}\left(B\left(x^{0}, \frac{\delta}{2}\right)\right) .
$$

Since $\nabla v=0$ on $B\left(x_{0}, \frac{\delta}{2}\right)$ (in fact, on $B\left(x_{0}, \frac{\delta}{2}\right)$, we have $\max \{R(2 \vartheta(x)-$ $1), u(x)\} \equiv R)$, it follows that $\left\langle\nabla_{\xi} L(\cdot, \nabla u(\cdot)),-\nabla u(\cdot)\right\rangle \in \mathrm{L}^{1}\left(B\left(x^{0}, \frac{\delta}{2}\right)\right)$; fix arbitrarily a compact $K \subset \Omega$; cover $K$ with finitely many balls $B\left(x^{i}, \frac{\delta^{i}}{2}\right)$ to obtain

$$
\left\langle\nabla_{\xi} L(\cdot, \nabla u(\cdot)), \nabla u(\cdot)\right\rangle \in \mathrm{L}_{\text {loc }}^{1}(\Omega) .
$$

b) In the special case where $L(x, \xi)=l(|\xi|)$, with $l$ a convex function having minimum at the origin, we have $\left\langle\nabla_{\xi} L(\xi), \xi\right\rangle=l^{\prime}(|\xi|)|\xi| \geq l^{\prime}(|\xi|)$ for $|\xi| \geq 1$ and the claim is proved.

In the general case, we can reason as follows. Let $x_{j}$ denote the $j$-th component of $x$. With the same notation as in a), let $R$ be so large that, for $1 \leq j \leq N$, we have $u(x)>-R+\left|x_{j}\right|$ on $B\left(x^{0}, 2 \delta\right)$ and $u(x)<R-\left|x_{j}\right|$ on $B\left(x^{0}, \frac{\delta}{2}\right)$.

Set

$$
v_{j}(x)= \begin{cases}\max \left\{R(2 \vartheta(x)-1)+x_{j}, u(x)\right\} & \text { if } x \in B\left(x^{0}, 2 \delta\right), \\ u(x) & \text { if } x \in \Omega \backslash B\left(x^{0}, 2 \delta\right),\end{cases}
$$

so that, on $B\left(x^{0}, \frac{\delta}{2}\right)$, we have $v_{j}(x)=R+x_{j}$ and $\nabla v_{j}=e_{j}$. An application of Lemma 2.4 yields that $\left\langle\nabla_{\xi} L(\cdot, \nabla u), e_{j}-\nabla u\right\rangle \in \mathrm{L}_{\text {loc }}^{1}$. Since, from 2.11, we have $\left\langle\nabla_{\xi} L(\cdot, \nabla u),-\nabla u\right\rangle \in \mathrm{L}_{\text {loc }}^{1}$, we infer that $\left\langle\nabla_{\xi} L(\cdot, \nabla u), e_{j}\right\rangle \in \mathrm{L}_{\text {loc }}^{1}$. 
Hence, $\left|\nabla_{\xi} L(\cdot, \nabla u)_{j}\right| \in \mathrm{L}_{\text {loc }}^{1}$. Since this is true for every $j$, we obtain that $\left|\nabla_{\xi} L(\cdot, \nabla u)\right| \in \mathrm{L}_{\text {loc }}^{1}$.

In particular, consider $L(\xi)=e^{|\xi|^{2}}$ so that $\nabla L(\xi)=2 e^{|\xi|^{2}} \xi$; let $u$ be a solution to the corresponding minimization problem. Then, by Lemma 2.5. we obtain

$$
\int_{\Omega} 2 e^{|\nabla u(x)|^{2}}|\nabla u(x)| d x<\infty .
$$

In the first part of 2 . we have presented an example with $\Omega=I=[0, b]$ and $v$ a locally bounded function such that $\int_{I} e^{\left|v^{\prime}(x)\right|^{2}} d x<+\infty$ but satisfying $\int_{I} e^{\left|v^{\prime}(x)\right|^{2}}\left|v^{\prime}(x)\right| d x=+\infty$; from Lemma 2.5 we can conclude that such function $v$ cannot possibly be a solution to a minimization problem with the given Lagrangian.

\section{A case of extended-valued Lagrangian and variational inequalities}

Consider the problem of minimizing

$$
\int_{\Omega}\left[\frac{1}{2}|\nabla v(x)|^{2}+v(x)\right] d x
$$

on the (convex) subset of those Lipschitzian functions with Lipschitz constant at most 1 that are null at $\partial \Omega$. Equivalently, we can introduce the extendedvalued, lower-semicontinuous and convex function $L_{\infty}$

$$
L_{\infty}(\xi)= \begin{cases}\frac{1}{2}|\xi|^{2} & \text { for }|\xi| \leq 1 \\ +\infty & \text { otherwise }\end{cases}
$$

and minimize

$$
\int_{\Omega}\left[L_{\infty}(\nabla v(x)+v(x)] d x \quad \text { on } \quad W_{0}^{1,1}(\Omega) .\right.
$$

The function $L_{\infty}$ is not differentiable when $|\xi|=1$. Still, the ideas of the previous lemmas can be applied. Let $u$ be a solution, let $v \in W_{0}^{1,1}(\Omega)$ be a function giving the finite value to the integral, so that $L_{\infty}(\nabla v(x))$ is a.e. finite. We know that

$$
\frac{1}{t}\left(L_{\infty}(\nabla u(x)+t(\nabla v(x)-\nabla u(x)))-L_{\infty}(\nabla u(x))\right)
$$


is a monotonic increasing function of $t$, so that a pointwise limit $p(x)$ exists as $t \downarrow 0$; since, for $0 \leq t \leq 1$ we have $\mid(\nabla u(x)+t(\nabla v(x)-\nabla u(x)) \mid \leq 1$, it follows that $L_{\infty}(\nabla u(x)+t(\nabla v(x)-\nabla u(x)))=\frac{1}{2}|\nabla u(x)+t(\nabla v(x)-\nabla u(x))|^{2}$ and $p(x)=\langle\nabla u(x), \nabla v(x)-\nabla u(x)\rangle$. As in (2.8), we have

$$
\begin{aligned}
\frac{1}{t}\left(L_{\infty}(\nabla u(x)+t(\nabla v(x)-\nabla u(x)))-\right. & \left.L_{\infty}(\nabla u(x))\right)^{+} \\
& \leq\left(L_{\infty}(\nabla v(x))-L_{\infty}(\nabla u(x))\right)^{+},
\end{aligned}
$$

and the right hand side is integrable. Then, by dominated convergence we obtain

$\int_{\Omega^{+}} p(x) d x=\lim _{t \rightarrow 0^{+}} \int_{\Omega^{+}} \frac{1}{t}\left(L_{\infty}(\nabla u(x)+t(\nabla v(x)-\nabla u(x)))-L_{\infty}(\nabla u(x))\right)^{+} d x$.

On the other hand, by Fatou's Lemma we infer

$$
\begin{gathered}
\int_{\Omega^{-}}-p(x) d x=\int_{\Omega^{-}} \lim _{t \rightarrow 0^{+}} \frac{1}{t}\left(L_{\infty}(\nabla u(x)+t(\nabla v(x)-\nabla u(x)))-L_{\infty}(\nabla u(x))\right)^{-} d x \\
\leq \liminf _{t \downarrow 0} \int_{\Omega^{-}} \frac{1}{t}\left(L_{\infty}(\nabla u(x)+t(\nabla v(x)-\nabla u(x)))-L_{\infty}(\nabla u(x))\right)^{-} d x
\end{gathered}
$$

and hence,

$\int_{\Omega^{-}} p(x) d x \geq \limsup _{t \downarrow 0} \int_{\Omega^{-}}-\frac{1}{t}\left(L_{\infty}(\nabla u(x)+t(\nabla v(x)-\nabla u(x)))-L_{\infty}(\nabla u(x))\right)^{-} d x$.

From the minimality condition we have

$$
\int_{\Omega^{+} \cup \Omega^{-}} \frac{1}{t}\left(L_{\infty}(\nabla u+t(\nabla v-\nabla u))-L_{\infty}(\nabla u)\right) d x+\int_{\Omega}(v-u) d x \geq 0
$$

so that

$$
\begin{aligned}
\int_{\Omega}[\langle\nabla u(x), \nabla v(x)-\nabla u(x)\rangle+(v-u)] d x & \\
\geq \limsup _{t \downarrow 0} \int_{\Omega}\left[\frac { 1 } { t } \left(L_{\infty}(\nabla u(x)+t(\nabla v(x)-\nabla u(x)))\right.\right. & \\
& \left.\left.-L_{\infty}(\nabla u(x))\right)+(v(x)-u(x))\right] d x \geq 0 .
\end{aligned}
$$


We have obtained a necessary condition in the form of a variational inequality: the solution $u$ satisfies: for every $v$ satisfying the boundary condition and giving a finite value to the integral, we have

$$
\int_{\Omega}[\langle\nabla u(x), \nabla v(x)-\nabla u(x)\rangle+(v(x)-u(x))] d x \geq 0 .
$$

\section{The Euler-Lagrange equation for problems with fast growth}

The Euler-Lagrange equation is an equality satisfied by variations $\eta$, independently on whether $u+\eta$ gives a finite value to the integral or not. The following is a form of the Euler-Lagrange equation for problems with fast growth. Notice that the conditions on $L$ imply that for a.e. $x$, the map $\xi \rightarrow L(x, \xi)$ takes finite values for every $\xi \in \mathbb{R}^{N}$, hence the next theorem does not apply to the functional of the previous section.

TheOREM 2.6. Let $L$ be as in Lemma 2.5. Let a solution $u$ to the problem of minimizing 2.5 be locally bounded. Then, for arbitrary $\eta \in C_{c}^{1}(\Omega)$, we have

$$
\int_{\Omega}\langle\nabla L(x, \nabla u(x)), \nabla \eta(x)\rangle d x=0 .
$$

Proof. Fix $\eta$ arbitrarily in $C_{c}^{1}(\Omega)$; choose $R$ so large that $-R<u(x)<R$ for $x \in \operatorname{supp}(\eta)$. For $t>1$ set

$$
\bar{v}_{t}(x)= \begin{cases}\max \{\operatorname{t\eta }(x)-R, u(x)\} & \text { if } x \in \operatorname{supp}(\eta), \\ u(x) & \text { otherwise }\end{cases}
$$

and

$$
\underline{v}_{t}(x)= \begin{cases}\min \{t \eta(x)+R, u(x)\} & \text { if } x \in \operatorname{supp}(\eta), \\ u(x) & \text { otherwise. }\end{cases}
$$

We have

$$
\begin{aligned}
& \nabla \bar{v}_{t}(x)= \begin{cases}\nabla u(x) & \text { if } u \geq t \eta-R \text { or } x \in \Omega \backslash \operatorname{supp}(\eta), \\
t \nabla \eta(x) & \text { otherwise, }\end{cases} \\
& \nabla \underline{v}_{t}(x)= \begin{cases}\nabla u(x) & \text { if } u \leq t \eta+R \text { or } x \in \Omega \backslash \operatorname{supp}(\eta), \\
t \nabla \eta(x) & \text { otherwise, }\end{cases}
\end{aligned}
$$


so that $L\left(\nabla \bar{v}_{t}\right)$ and $L\left(\nabla \underline{v}_{t}\right)$ belong to $L^{1}(\Omega)$. Applying Lemma 2.4 to these variations we obtain

$$
\left\langle\nabla L(\cdot, \nabla u), \nabla\left(\bar{v}_{t}-u\right)\right\rangle \in \mathrm{L}^{1}(\Omega) ; \quad \int_{\Omega}\left\langle\nabla_{\xi} L(x, \nabla u), \nabla\left(\bar{v}_{t}-u\right)\right\rangle d x \geq 0
$$

and

$$
\left\langle\nabla_{\xi} L(\cdot, \nabla u), \nabla\left(\underline{v}_{t}-u\right)\right\rangle \in \mathrm{L}^{1}(\Omega) ; \quad \int_{\Omega}\left\langle\nabla_{\xi} L(x, \nabla u), \nabla\left(\underline{v}_{t}-u\right)\right\rangle d x \geq 0 .
$$

For $t>1$ consider the set $A_{t}=\left\{\eta>\frac{u+R}{t}\right\} \cap \operatorname{supp}\left(\eta^{+}\right)$; since $u>-R$ on $\operatorname{supp}\left(\eta^{+}\right)$, we have $\eta(x)>0$ for $x \in A_{t}$, hence that $\nabla \eta=\nabla\left(\eta^{+}\right)$there. Moreover, $t \eta-R>u$ in $A_{t}$, so that, for $x$ in $A_{t}$, we have $\bar{v}_{t}=t \eta-R$ while, in $\Omega \backslash A_{t}, \bar{v}_{t}-u=0$ and $\nabla\left(\bar{v}_{t}-u\right)=0$. Then, dividing by $t$, 2.13) yields

$$
\begin{aligned}
& \int_{\Omega}\left\langle\nabla_{\xi} L(x, \nabla u), \nabla\left(\bar{v}_{t}-u\right)\right\rangle d x=\int_{A_{t}}\left\langle\nabla_{\xi} L(x, \nabla u), \nabla \eta-\frac{1}{t} \nabla u\right\rangle d x \\
&=\int_{\Omega} \chi_{A_{t}}(x)\left\langle\nabla_{\xi} L(x, \nabla u), \nabla \eta-\frac{1}{t} \nabla u\right\rangle d x \geq 0 .
\end{aligned}
$$

The function $t \rightarrow \chi_{A_{t}}(x)$ is non-decreasing, hence pointwise converging to $\chi_{\operatorname{supp}\left(\eta^{+}\right)}(x)$; then, as $t \rightarrow+\infty$, we infer that $\chi_{A_{t}}(x)\left\langle\nabla_{\xi} L(x, \nabla u(x)), \nabla \eta(x)-\right.$ $\left.\frac{1}{t} \nabla u(x)\right\rangle$ converges pointwise to $\chi_{\operatorname{supp}\left(\eta^{+}\right)}(x)\left\langle\nabla_{\xi} L(x, \nabla u(x)), \nabla\left(\eta^{+}\right)(x)\right\rangle$.

Let $H=\sup \{|\nabla \eta(x)|\}$. We have

$$
\left|\chi_{A_{t}}\left\langle\nabla_{\xi} L(x, \nabla u), \nabla \eta-\frac{1}{t} \nabla u\right\rangle\right| \leq H\left|\nabla_{\xi} L(x, \nabla u)\right|+\left|\left\langle\nabla_{\xi} L(x, \nabla u), \nabla u\right\rangle\right| .
$$

By Lemma 2.5 both $\left\langle\nabla_{\xi} L(x, \nabla u), \nabla u\right\rangle$ and $\left|\nabla_{\xi} L(x, \nabla u)\right|$ are in $\mathrm{L}_{\text {loc }}^{1}(\Omega)$. By dominated convergence we conclude that

$$
\begin{aligned}
& 0 \leq \lim _{t \rightarrow+\infty} \int_{A_{t}}\left\langle\nabla L(x, \nabla u), \nabla \eta-\frac{1}{t} \nabla u\right\rangle d x \\
& =\int_{\Omega} \chi_{\operatorname{supp}\left(\eta^{+}\right)}\left\langle\nabla L(x, \nabla u), \nabla\left(\eta^{+}\right)\right\rangle d x=\int_{\Omega}\left\langle\nabla L(x, \nabla u), \nabla\left(\eta^{+}\right)\right\rangle d x .
\end{aligned}
$$

Analogously, consider the set $B_{t}=\left\{\eta<\frac{u-R}{t}\right\} \cap K$; we have $\underline{v}_{t}=t \eta+R$ in $B_{t}$. Through the same steps as before, we obtain

$$
\int_{\Omega}\left\langle\nabla L(x, \nabla u), \nabla\left(-\eta^{-}\right)\right\rangle d x \geq 0 .
$$


Add 2.14 to 2.15 to yield: for every $\eta \in C_{c}^{\infty}(\Omega)$,

$$
\int_{\Omega}\langle\nabla L(x, \nabla u), \nabla \eta\rangle d x \geq 0 .
$$

Finally, consider $\tilde{\eta}=-\eta$ : 2.16 yields

$$
\int_{\Omega}\langle\nabla L(x, \nabla u), \nabla \eta\rangle d x \leq 0
$$

so that

$$
\int_{\Omega}\langle\nabla L(x, \nabla u), \nabla \eta\rangle d x=0 .
$$

REMARK. In a previous section we have pointed out that, given $\eta$, in order to pass to the limit as $\varepsilon_{n} \rightarrow 0$ in

$$
\int_{\Omega} \frac{L\left(\nabla u(x)+\varepsilon_{n} \nabla \eta(x)\right)-L(\nabla u(x))}{\varepsilon_{n}} d x,
$$

in order to obtain

$$
\int_{\Omega}\langle\nabla L(x, \nabla u(x)), \nabla \eta(x)\rangle d x=0
$$

we need to know that both $L(\cdot, \nabla u(\cdot)+\nabla \eta(\cdot))$ and $\left|\left\langle\nabla_{\xi} L(\cdot, \nabla u(\cdot)), \nabla \eta(\cdot)\right\rangle\right|$ are integrable on $\Omega$. Lemma 2.5 assures (under the conditions of validity of the lemma, but without upper growth conditions) the integrability of the second; Theorem 2.6 above assures that, for arbitrary $\eta$, equation 2.17 holds. Can we then, finally, obtain the validity of equation (2.17) by dominated convergence?

By no means. Theorem 2.6 does not assure that there exists even one non-trivial $\eta \in C_{c}^{1}(\Omega)$ such that $L(\cdot, \nabla u(\cdot)+\nabla \eta(\cdot)) \in \mathrm{L}^{1}(\Omega)$.

If by the name of variation we mean a smooth function $\eta$, that is zero at the boundary of $\Omega$ and gives a finite value to the integral $\int_{\Omega} L(x, \nabla u(x)+$ $\nabla \eta(x)) d x$, then, in general, variations need not exist. Still, remarkably, for the functions $L$ considered in this paragraph, the Euler-Lagrange equation holds.

REMARK. For some purposes, in particular to obtain regularity estimates for the solution, results on the validity of the Euler-Lagrange equation in a form stronger than the one presented in Theorem 2.6, i.e., when the variations are not necessarily continuously differentiable, is needed. A typical variation used in these estimates contains some derivatives of the solution, and there is no reason to expect that these derivatives are smooth. However, the proof of Theorem 2.6 depends on being $\nabla \eta$ bounded. 
In [5] the following stronger form of Theorem 2.6 is proved.

TheOREM 2.7. Let $L$ and $u$ be as in the statement of Theorem 2.6 and, in addition, let $L(0)=0, \nabla L(0)=0$ and $L(\xi)=L(-\xi)$; let $w \in W^{1,1}(\Omega)$ be such that $\operatorname{supp}(w) \subset \subset \Omega$ and such that, for some $\alpha \in(0,1]$ we have $\int_{\Omega} L(\alpha \nabla w)<$ $\infty$. Then, $\int_{\Omega}\langle\nabla L(\nabla u), \nabla w\rangle=0$.

A variation $w \in C_{c}^{1}(\Omega)$ satisfies the assumptions of the previous theorem, as does the variation $w=\delta_{-h}^{i}\left(\eta^{2} \delta_{h}^{j} u\right)$, where $\eta \in C_{c}^{1}(\Omega)$. This variation is mostly used in regularity proofs.

An open problem. Consider the problem of minimizing

$$
\int_{\Omega} L(|\nabla u(x)|) d x
$$

where

$$
L(t)=\frac{1}{1-t^{2}}
$$

for $|t|<1$, and $L(t)=+\infty$ otherwise.

$L$ is convex, differentiable; the solution $\bar{u}$ to the minimization problem (with suitable boundary conditions) exists and is unique; moreover, $\bar{u}$ is Lipschitzian with Lipschitz constant 1 and hence $\bar{u}$ is locally bounded.

We have $L^{\prime}(t)=2 \frac{t}{\left(1-|t|^{2}\right)^{2}}$ so that the Euler-Lagrange equation would be:

$$
\int_{\Omega} \frac{1}{\left(1-|\nabla \bar{u}(x)|^{2}\right)^{2}}\langle\nabla \bar{u}(x), \nabla \eta(x)\rangle d x=0
$$

for every $\eta \in C_{c}^{1}(\Omega)$.

The author has tried for long, but was unable to prove its validity.

In this case, the domain of $L$ is an open convex interval different from $\mathbb{R}^{N}$.

\section{Lagrangians that are convex but not necessarily differentiable}

The convexity of $L$ with respect to the variable $\xi$ is a condition generally considered minimal in the Calculus of Variations. Convexity in itself has a form of differentiability, the subdifferential, without the need of extra regularity. The purpose of this section is to explore to what an extent the condition of convexity by itself yields a form of the Euler-Lagrange equation. 
EXAmple. Let $\Omega$ be a bounded open subset of $\mathbb{R}^{2}$, containing the ball $B(0,2 \sqrt{2})$, and consider the minimization problem

$$
\operatorname{minimize} \quad \int_{\Omega}[F(\nabla v(x))+v(x)] d x
$$

where

$$
F(\xi)=f(\|\xi\|)= \begin{cases}\sqrt{2}\|\xi\| & \text { for }\|\xi\| \leq \sqrt{2} \\ 1+\frac{1}{2}\|\xi\|^{2} & \text { for }\|\xi\| \geq \sqrt{2}\end{cases}
$$

We have $\partial F(0)=\sqrt{2} B[0,1]$ while $\nabla F(\xi)=\xi$ for $\xi \neq 0$. Consider the function $u(x)=\min \left\{0,\left(\frac{\|x\|}{2}\right)^{2}-2\right\}$, i.e.,

$$
u(x)= \begin{cases}0 & \text { for } \frac{\|x\|}{2} \leq \sqrt{2} \\ \left(\frac{\|x\|}{2}\right)^{2}-2 & \text { for } \frac{\|x\|}{2} \geq \sqrt{2}\end{cases}
$$

so that

$$
\nabla u(x)=\left\{\begin{array}{cc}
0 & \text { for } \frac{\|x\|}{2}<\sqrt{2} \\
\frac{1}{2} x & \text { for } \frac{\|x\|}{2}>\sqrt{2} .
\end{array}\right.
$$

We claim that it is a solution to the minimization problem, among the functions $v$ satisfying the same values as $u$ on $\partial \Omega$, i.e., more precisely, for $v \in$ $u+W_{0}^{1,1}(\Omega)$.

We obtain

$$
\partial F(\nabla u(x))= \begin{cases}\sqrt{2} B[0,1] & \text { for } \frac{\|x\|}{2}<\sqrt{2}, \\ \nabla F(\nabla u(x))=\nabla \tilde{u}(x)=\frac{1}{2} x & \text { for } \frac{\|x\|}{2}>\sqrt{2} .\end{cases}
$$

On $\omega$ consider also the smooth function

$$
p(x)=\frac{1}{2} x
$$

and notice that, for every $x$, we have $p(x) \in \partial F(\nabla u(x))$. Since $\operatorname{div}(p(x))=1$, we have, for every $\eta \in C_{c}^{\infty}$,

$$
\int_{\Omega}[\langle p(x), \nabla \eta(x)\rangle+\eta(x)] d x=0 .
$$


The map $G:(v, \xi) \rightarrow F(\xi)+v$ is convex and, when $p \in \partial F(\xi)$, also $(1, p) \in$ $\partial G((v, \xi))$ so that

$$
\begin{aligned}
& G(u(x)+\eta(x), \nabla u(x)+\nabla\eta(x))=F(\nabla u(x)+\nabla \eta(x))+u(x)+\eta(x) \\
& \geq F(\nabla u(x))+u(x)+1 \cdot \eta(x)+\langle p(x), \nabla \eta(x)\rangle .
\end{aligned}
$$

Hence

$$
\begin{aligned}
\int_{\Omega}(F(\nabla u(x) & +\nabla \eta(x))+u(x)+\eta(x)) d x \geq \int_{\Omega}(F(\nabla u(x))+u(x)) d x \\
+ & \int_{\Omega}(1 \cdot \eta(x)+\langle p(x), \nabla \eta(x)\rangle) d x=\int_{\Omega}(F(\nabla u(x))+u(x)) d x .
\end{aligned}
$$

The usual approximation scheme shows that

$$
\int_{\Omega}(F(\nabla u(x)+\nabla \eta(x))+u(x)+\eta(x)) d x \geq \int_{\Omega}(F(\nabla u(x))+u(x)) d x
$$

for every $\eta \in W_{0}^{1,2}$, hence $u$ is a solution.

Although the function $\nabla \tilde{u}(x)$ is discontinuous, the vector function

$$
p(x)=\frac{1}{2} x
$$

is an everywhere smooth selection from the map $x \rightarrow \partial F(\nabla u(x))$ and has the divergence equal to 1 everywhere, so that the Euler-Lagrange equation is satisfied even when there is no differentiability assumption on the convex function $F$.

Assume that we are minimizing a functional of the kind

$$
\int_{\Omega}[L(\nabla v(x))+g(x, v(x))] d x
$$

where both $L$ and $v \rightarrow g(x, v)$ are convex. We assume that $g$ is differentiable, but we do not make such assumption on $L$. Then, by convexity, for any $u$ and $v$, we have, for any $p(x)$ in $\partial L(\nabla u(x))$

$$
\begin{aligned}
L(\nabla u(x))+g(x, u(x)) & \geq L(\nabla v(x))+g(x, v(x)) \\
+ & \langle p(x), \nabla u(x)-\nabla v(x)\rangle+g_{u}^{\prime}(x, u(x))(u(x)-v(x)) ;
\end{aligned}
$$


if, in addition, the map $x \rightarrow p(x)$ is regular enough to make the integral meaningful, we obtain

$$
\begin{aligned}
\int_{\Omega}[L(\nabla u(x)) & +g(x, u(x))] d x \geq \int_{\Omega}[L(\nabla v(x))+g(x, v(x))] d x \\
& +\int_{\Omega}\left[\langle p(x), \nabla u(x)-\nabla v(x)\rangle+g_{u}^{\prime}(x, u(x))(u(x)-v(x))\right] d x .
\end{aligned}
$$

If the second integral to the right, above, is zero, we have $\int_{\Omega}[L(\nabla u(x))+$ $g(x, u(x))] d x \geq \int_{\Omega}[L(\nabla v(x))+g(x, v(x))] d x$, i.e., that $u$ is a solution. Since $u$ and $v$ satisfy the same boundary conditions, setting $\eta(x)=u(x)-v(x), \eta$ is a variation and the condition on the integral is

$$
\int_{\Omega}\left[\langle p(x), \nabla \eta(x)\rangle+g_{u}^{\prime}(x, u(x)) \eta(x)\right] d x=0 .
$$

When $L$ is differentiable, so that $p(x)=\nabla L(\nabla u(x))$, the condition becomes

$$
\int_{\Omega}\left[\langle\nabla L(\nabla u(x)), \nabla \eta(x)\rangle+g_{u}^{\prime}(x, u(x)) \eta(x)\right] d x=0,
$$

i.e., the Euler-Lagrange equation. This suggests that, in the case of nondifferentiabilty of $L$, it would be of interest to prove the following form of the Euler-Lagrange equation: Let $u$ be a solution to the minimum problem; then, there exists a function $p(\cdot)$, a selection from the map $x \rightarrow \partial L(\nabla u(x))$ such that, for any $\eta$ in a suitable class of variations, we have

$$
\int_{\Omega}\left[\langle p(x), \nabla \eta(x)\rangle+g_{u}^{\prime}(x, u(x)) \eta(x)\right] d x=0 .
$$

To prove a result in this direction we shall need some language and notations from Convex Analysis (see, e.g., [15]).

The indicator function of a set $A, I_{A}(\xi)$, equals zero for $\xi \in A$, equals $+\infty$ elsewhere. Its polar is

$$
\left(I_{A}\right)^{*}(p)=\sup _{\xi \in \mathbb{R}^{N}}\left[\langle p, \xi\rangle-I_{A}(\xi)\right]=\sup _{\xi \in A}\langle p, \xi\rangle .
$$

When $A=B(0, h)$ ( $B$ the Euclidean ball) we have $\left(I_{A}\right)^{*}(p)=h|p|$.

Notice that the polar is always a convex function. Moreover, $\lambda$ non-negative implies that $\sup _{\xi \in A}\langle\lambda p, \xi\rangle=\lambda \sup _{\xi \in A}\langle p, \xi\rangle$, i.e., the map $p \rightarrow\left(I_{A}\right)^{*}(p)$ is positively homogeneous. 


\section{Riesz's Representation Theorem for continuous functionals acting on $L^{1}$}

LEMmA 2.8. Let $T$ be a bounded linear functional on $\left(\mathrm{L}^{1}(\Omega)\right)^{N}$, i.e. such that, for some $k$, we have

$$
T(\xi) \leq k\|\xi\|_{L^{1}}=k \int_{\Omega}|\xi(x)| d x=\int_{\Omega}\left(I_{k B(0,1)}\right)^{*}(\xi(x)) d x
$$

for every $\xi \in\left(\mathrm{L}^{1}(\Omega)\right)^{N}$. Then, there exists $\tilde{p} \in\left(\mathrm{L}^{\infty}(\Omega)\right)^{N}, \tilde{p}(x)$ a.e. in $k B(0,1)$, that represents $T$, i.e., such that

$$
T(\xi)=\int_{\Omega}\langle\tilde{p}(x), \xi(x)\rangle d x .
$$

A generalization. Notation: given a closed convex $K \subset \mathbb{R}^{N}$, by $m_{K}$ we mean the unique point of $K$ of minimal norm and by $\|K\|$ we mean $\sup \{|k|: k \in K\}$.

LEMMA 2.9. Let $D$ be a map from $\Omega$ to the closed convex non-empty subsets of $B(0, R)$, such that $\|D(\cdot)\| \in L^{\infty}$ and such that $v \in\left(\mathrm{L}^{\infty}(\Omega)\right)^{N}$ implies that the map $x \rightarrow m_{[D(x)-v(x)]}$ is measurable. Let $T:\left(\mathrm{L}^{1}(\Omega)\right)^{N} \rightarrow \mathbb{R}$ be a linear functional satisfying

$$
T(\xi) \leq \int_{\Omega}\left(I_{D(x)}\right)^{*}(\xi(x)) d x
$$

Then there exists $\tilde{p} \in\left(\mathrm{L}^{\infty}(\Omega)\right)^{N}, \tilde{p}(x)$ in $D(x)$ a.e., that represents $T$, i.e., such that 2.18 holds.

Proof of the Lemma. a) Since $\left|\left(I_{D(x)}\right)^{*}(\xi(x))\right| \leq\|D(x)\||\xi(x)|$ we have $T$ is a bounded linear functional on $\left(\mathrm{L}^{1}(\Omega)\right)^{N}$. Writing $\xi$ as $\xi_{1}(x) e_{1}+\ldots+$ $\xi_{N}(x) e_{N}$ and applying the standard Riesz's Representation Theorem, we infer the existence of a function $\tilde{p} \in\left(\mathrm{L}^{\infty}(\Omega)\right)^{N}$ that satisfies 2.18). To show that $\tilde{p}(x)$ is in $D(x)$ a.e., assume that there exists a set $E \subset \Omega$ of positive measure such that, on $E, \tilde{p}(x) \notin D(x)$, i.e. $0 \notin D(x)-\tilde{p}(x)$. Setting $D^{*}:=D(x)-\tilde{p}(x)$, we can equivalently say that $\left|m_{D^{*}(x)}\right|>0$ on $E$.

Let $z(x)$ be the projection of minimal distance of $\tilde{p}(x)$ on $D(x)$, so that, $z(x)-\tilde{p}(x)=m_{D(x)-\tilde{p}(x)}$ or, $z(x)-\tilde{p}(x)=m_{D^{*}(x)}$. From the characterization of the projection of minimal distance, we obtain

$$
\langle\tilde{p}(x)-z(x), z(x)\rangle \geq\langle\tilde{p}(x)-z(x), k\rangle, \quad \forall k \in D(x),
$$


that can be rewritten as

$$
\left\langle-m_{D^{*}}(x), \tilde{p}(x)\right\rangle \geq\left|m_{D^{*}}(x)\right|^{2}+\left\langle-m_{D^{*}}(x), k\right\rangle, \quad \forall k \in D(x) .
$$

Hence, we have, on $E$,

$$
\left\langle-m_{D^{*}}(x), \tilde{p}(x)\right\rangle>\sup \left\{\left\langle-m_{D^{*}}(x), k\right\rangle: k \in D(x)\right\}=\left(I_{D(x)}\right)^{*}\left(-m_{D^{*}}(x)\right) .
$$

b) Setting $\tilde{\xi}:=-m_{D^{*}} \chi_{E}$, we have $\tilde{\xi} \in \mathrm{L}^{1}(\Omega)$ and

$$
T(\tilde{\xi})=\int_{\Omega}\langle\tilde{p}, \tilde{\xi}\rangle=\int_{E}\left\langle\tilde{p},-m_{D^{*}}\right\rangle>\int_{\Omega}\left(I_{D(x)}\right)^{*}(\tilde{\xi}) \geq T(\tilde{\xi}),
$$

a contradiction.

Idea of the proof of the validity of the Euler-Lagrange equation without differentiability conditions w.r. to $\xi$.

Consider the simple problem of minimizing

$$
\int_{\Omega}[L(\nabla u(x))+g(u(x))] \mathrm{d} x
$$

where $L$ is convex but not necessarily differentiable. $L$ is defined everywhere, so that $\partial L(\xi)$ exists non-empty everywhere; $g$ is differentiable.

Let $u$ be a locally bounded solution to problem 2.19$)$, let $\eta \in C_{0}^{\infty}(\Omega)$. Set $D_{L}(x)=\partial_{\xi} L(\nabla u(x))$; we have

$$
\begin{aligned}
\frac{1}{\varepsilon}[L(\nabla u(x)+\varepsilon \nabla \eta(x))-L(\nabla u(x)) & +g(u(x)+\varepsilon \eta(x))-g(u(x))] \\
& \rightarrow\left[\sup _{k \in D_{L}(x)}\langle k, \nabla \eta(x)\rangle\right]+g^{\prime}(u(x)) \eta(x)
\end{aligned}
$$

pointwise w.r.t. $x$. With the notation we have introduced, we write

$$
\sup _{k \in D_{L}(x)}\langle k, \nabla \eta(x)\rangle=\left(I_{D_{L}(x)}\right)^{*}(\nabla \eta(x))
$$

Assume that we have (growth) conditions that allow us to pass to the limit under integral sign. Hence, passing to the limit, we obtain

$$
0 \leq \int_{\Omega}\left(I_{D_{L}(x)}\right)^{*}(\nabla \eta(x)) \mathrm{d} x+\int_{\Omega} g^{\prime}(u(x)) \eta(x) \mathrm{d} x .
$$


Our purpose is to find an integrable function $p$ with two properties:

i) a.e. $p(x) \in D_{L}(x)=\partial_{\xi} L(\nabla u(x))$;

ii) for every $\eta \in C_{0}^{\infty}(\Omega)$, we must have

$$
\int_{\Omega}\langle p(x), \nabla \eta(x)\rangle \mathrm{d} x+\int_{\Omega} g^{\prime}(u(x)) \eta(x) \mathrm{d} x=0 .
$$

When $L$ is differentiable, so that $\partial_{\xi} L=\nabla L$, this is the usual Euler-Lagrange equation.

The difficulty arises from the fact that we are not able to define $p$ pointwise: $p(x)$ is not the pointwise limit of $\frac{1}{\varepsilon}[L(\nabla u(x)+\varepsilon \nabla \eta(x))-L(\nabla u(x))]$.

The idea of the proof comes from looking at equation (2.21) in this other form: we have to find $p$ such that

$$
\int_{\Omega}\langle p(x), \nabla \eta(x)\rangle \mathrm{d} x=-\int_{\Omega} g^{\prime}(u(x)) \eta(x) \mathrm{d} x .
$$

What we have at the r.h.s. is certainly a linear functional on $\eta$; hence, we have to find $p$ such that the l.h.s. represents this linear functional.

a) Rewrite 2.20 as

$$
-\int_{\Omega} g^{\prime}(u(x)) \eta(x) \mathrm{d} x \leq \int_{\Omega}\left(I_{D_{L}(x)}\right)^{*}(\nabla \eta(x)) \mathrm{d} x
$$

so that the r.h.s. is an upper bound for the linear functional at the left (similar to what happens in Riesz's Theorem).

One technical difficulty: Riesz's Theorem represents a functional acting on $L^{1}$ and gives a representative in the dual $L^{\infty}$. Here we have a functional on $\eta$ (that is in $L^{\infty}$ ) and we seek a $p$ to represent it, with $p \in L^{1}$, since $p \in D_{L}(x)=$ $\partial_{\xi} L(\nabla u(x))$ that can be very large. To avoid this difficulty, it is convenient to look at the problem in a different way: set $\|D\|=\sup \{|v|: v \in D\}$; then we have

$$
\begin{aligned}
\left(I_{D_{L}(x)}\right)^{*}(\nabla \eta) & =\sup _{k \in D_{L}(x)}\langle k, \nabla \eta\rangle=\sup _{k \in D_{L}(x)}\left\langle\frac{k}{\left\|D_{L}(x)\right\|},\left\|D_{L}(x)\right\| \nabla \eta\right\rangle \\
& =\sup _{k \in \frac{D_{L}(x)}{\left\|D_{L}(x)\right\|}}\left\langle k,\left\|D_{L}(x)\right\| \nabla \eta\right\rangle=\left(I_{\frac{D_{L}(x)}{\left\|D_{L}(x)\right\|}}\right)^{*}\left(\left\|D_{L}(x)\right\| \nabla \eta\right)
\end{aligned}
$$

and inequality 2.22 becomes

$$
-\int_{\Omega} g^{\prime}(u(x)) \eta(x) \mathrm{d} x \leq \int_{\Omega}\left(I_{\frac{D_{L}(x)}{\left\|D_{L}(x)\right\|}}\right) *\left(\left\|D_{L}(x)\right\| \nabla \eta\right) \mathrm{d} x .
$$


Notice that the map

$$
\varrho(\xi):=\int_{\Omega}\left(I_{\frac{D_{L}(x)}{\left\|D_{L}(x)\right\|}}\right)^{*}(\xi(x)) \mathrm{d} x
$$

is well defined for every $\xi \in\left(L^{1}(\Omega)\right)^{N}$, since $\left(I_{\frac{D_{L}(x)}{\left\|D_{L}(x)\right\|}}\right)^{*}(\xi) \leq|\xi|$, and that it is a convex and positively homogeneous map. Hence we have a linear functional $\int_{\Omega} g^{\prime}(u(x)) \eta(x) \mathrm{d} x$, defined on some subspace, bounded from above by a convex and positively homogeneous map $\varrho$, defined over the whole of $\left(L^{1}(\Omega)\right)^{N}$.

b) From the growth conditions (for instance, a polynomial growth) we obtain that $\left\|D_{L}\right\| \in \mathrm{L}^{1}(\Omega)$; for every $\eta \in C_{0}^{\infty}(\Omega)$ we have $\left\|D_{L}\right\| \nabla \eta \in\left(\mathrm{L}^{1}(\Omega)\right)^{N}$. Consider $\mathbb{L}$, the linear subspace of $\left(\mathrm{L}^{1}(\Omega)\right)^{N}$, defined as

$$
\mathbb{L}=\left\{\xi \in\left(L^{1}(\Omega)\right)^{N}: \exists \eta \in C_{0}^{\infty}(\Omega): \xi=\left\|D_{L}(x)\right\| \nabla \eta\right\}
$$

and, on $\mathbb{L}$, the linear functional

$$
T(\xi)=-\int_{\Omega} g^{\prime}(u(x)) \eta(x) \mathrm{d} x .
$$

We notice that $T$ is well defined: assume that there exist $\eta^{1}$ and $\eta^{2}$ in $C_{0}^{\infty}(\Omega)$ such that $\frac{\xi}{\left\|D_{L}\right\|}=\nabla \eta^{1}=\nabla \eta^{2}$; then, by Poincaré's inequality, $\eta^{1}=\eta^{2}$.

By the Hahn-Banach Theorem, the linear map $T$ can be extended, as $\tilde{T}$, from $\mathbb{L}$ to the whole of $\left(\mathrm{L}^{1}(\Omega)\right)^{N}$, still satisfying $\tilde{T}(\xi) \leq \rho(\xi)$.

c) At this point, we prove that the map $x \rightarrow \partial_{\xi} L(\nabla u(x))$ satisfies the measurability conditions required by Lemma 2 , and so does the map $x \rightarrow \frac{1}{\left\|D_{L}\right\|} D_{L}$. Hence, we infer the existence of a $\tilde{p} \in\left(\mathrm{L}^{\infty}(\Omega)\right)^{N}$, with $\tilde{p}(x) \in \frac{1}{\left\|D_{L}(x)\right\|} D_{L}(x)$ a.e. on $\Omega$, i.e., $\tilde{p}(x)=\frac{1}{\left\|D_{L}(x)\right\|} p(x)$ with $p(x) \in D_{L}(x)$, representing the extension of $T$ to $\left(\mathrm{L}^{1}(\Omega)\right)^{N}$, in particular, representing $T$ on $\mathbb{L}$. Hence, for every $\eta \in C_{0}^{\infty}(\Omega)$, we have

$$
-\int_{\Omega} g^{\prime}(u(x)) \eta(x) \mathrm{d} x=\int_{\Omega}\left\langle\tilde{p}(x),\left\|D_{L}(x)\right\| \nabla \eta(x)\right\rangle \mathrm{d} x=\int_{\Omega}\langle p(x), \nabla \eta(x)\rangle \mathrm{d} x .
$$

In other words, for every $\eta \in C_{0}^{\infty}(\Omega)$,

$$
\int_{\Omega}\langle p(x), \nabla \eta(x)\rangle \mathrm{d} x+\int_{\Omega} g^{\prime}(u(x)) \eta(x) \mathrm{d} x=0 .
$$


The map $p(\cdot)$ is a selection from $\partial_{\xi} L(\nabla u(\cdot))$ defined on $\Omega$, thus proving the validity of the Euler-Lagrange equation.

REMARK. As a case of a Lagrangian that is convex but not differentiable we have the map $L_{\infty}$ defined before. The results of this section do not apply to this Lagrangian, since there are no upper growth conditions to be exploited to give an upper $L^{1}$ bound for $p$. Still, a form of the Euler-Lagrange equation for the problem (2.12) has been proved by Brézis [1]. However, the proof depends on regularity assumptions made on $\partial \Omega$; a general proof, based on local regularity, is still to be found.

\section{Existence and non-existence of solutions for Variational Problems of slow (linear) growth}

As it is well known, the existence of solutions to a minimum problem of the Calculus of Variations is based on the two concepts of convexity and coercivity. This last property is obtained imposing superlinear growth conditions on the integrand, hence it requires integrands $L$ that grow more than linearly in the variable $\nabla v$. A famous example that does not satisfy this condition is provided by the non-parametric minimal area problem, where we wish to minimize the functional

$$
\int_{\Omega} \sqrt{1+|\nabla u(x)|^{2}} d x
$$

under assigned boundary conditions.

\section{A classical example of non-existence of solutions}

Consider in dimension two the annulus $A=\left\{x: \frac{1}{2} \leq|x| \leq 1\right\}$. We plan to show that for $\alpha$ sufficiently large, the problem of minimizing

$$
\int_{A} \sqrt{1+|\nabla u(x)|^{2}} d x
$$

with the boundary condition

$$
u(x)=0 \text { for }|x|=1 ; \quad u(x)=-\alpha \text { for }|x|=\frac{1}{2}
$$

admits no solution. 
Proof of this Claim. a) We begin by looking for radial solutions. The Euler-Lagrange equation gives

$$
\frac{d}{d r} r \frac{u^{\prime}(r)}{\sqrt{1+\left(u^{\prime}(r)\right)^{2}}}=0
$$

and hence that $r \frac{u^{\prime}(r)}{\sqrt{1+\left(u^{\prime}(r)\right)^{2}}}=c$. Since, for some point in $\left(\frac{1}{2}, 1\right)$, we must have $u^{\prime}(r)>0$, then $c>0$ and we infer that $u^{\prime}(r)>0$ on $\left(\frac{1}{2}, 1\right)$. We have $r u^{\prime}(r)=$ $c \sqrt{1+\left(u^{\prime}(r)\right)^{2}}$; since $\sqrt{1+\left(u^{\prime}(r)\right)^{2}}>u^{\prime}(r)$, we must have $r>c$, for every $r$ in $\left(\frac{1}{2}, 1\right)$. Squaring, we obtain $\left(u^{\prime}(r)\right)^{2}\left(r^{2}-c^{2}\right)=c^{2}$, i.e., $u^{\prime}(r)=\frac{c}{\sqrt{r^{2}-c^{2}}}$, that gives $u(r)=\int_{1}^{r} \frac{1}{\sqrt{\frac{r^{2}}{c^{2}}-1}} d r=\int_{\frac{c}{c}}^{\frac{r}{c}} \frac{c}{\sqrt{y^{2}-1}} d y$. We obtain

$$
u(r)=\left.c \ln \left(y+\sqrt{y^{2}-1}\right)\right|_{\frac{1}{c}} ^{\frac{r}{c}}=c \ln \frac{r+\sqrt{r^{2}-c^{2}}}{1+\sqrt{1-c^{2}}} .
$$

Since $c \geq \frac{1}{2}$, the denominator inside the logarithm is larger than $1+\frac{\sqrt{3}}{2}$ and the numerator is smaller than 2 so that

$$
|u(r)| \leq \frac{1}{2}\left|\ln \left(\frac{2}{1+\frac{\sqrt{3}}{2}}\right)\right| .
$$

In particular, so is $\left|u\left(\frac{1}{2}\right)\right|$ and there is no way it can equal $|\alpha|$ if $|\alpha|$ is large.

b) Let us show that the problem does not admit (possibly non-radial) solutions.

We need the following proposition, taken from [8]:

Proposition 3.1. Let $u \in W^{1,1}(A)$. For $x \in A$ write $x=\omega|x|$ with $|\omega|=1$ and set

$$
\tilde{u}(x)=\frac{1}{2 \pi} \int_{|\omega|=1} u(\omega|x|) d \omega .
$$

Then

$$
\nabla \tilde{u}(x)= \begin{cases}\frac{1}{2 \pi} \frac{x}{|x|} \int_{|\omega|=1}\langle\nabla u(\omega|x|), \omega\rangle d \omega & \text { if } x \neq 0 \\ 0 & \text { if } x=0\end{cases}
$$


Assuming this proposition, let $u \in W^{1,1}(A)$ be a (non-radial) solution to the problem of minimizing (3.1) with the given boundary conditions, and $|\alpha|$ larger than $\frac{1}{2}\left|\ln \left(\frac{2}{1+\frac{\sqrt{3}}{2}}\right)\right|$; set $\tilde{u}$ be defined as in the statement of Proposition 3.1. Then, $\tilde{u}$ satisfies the same (radial) boundary conditions as $u$. We wish to show that

$$
\int_{A} l(|\nabla \tilde{u}(x)|) d x \leq \int_{A} l(|\nabla u(x)|) d x .
$$

From Proposition 3.1, we infer that

$$
|\nabla \tilde{u}(x)| \leq \frac{1}{2 \pi} \int_{|\omega|=1}|\nabla u(\omega|x|)| d \omega
$$

and, since the map $t \rightarrow l(t)=\sqrt{1+t^{2}}$ is increasing for $t \geq 0$, we obtain that

$$
l(|\nabla \tilde{u}(x)|) \leq l\left(\frac{1}{2 \pi} \int_{|\omega|=1}|\nabla u(\omega|x|)| d \omega\right) .
$$

Jensen's inequality gives

$$
l(|\nabla \tilde{u}(x)|) \leq \frac{1}{2 \pi} \int_{|\omega|=1} l(|\nabla u(\omega|x|)|) d \omega
$$

and hence

$$
\int_{A} l(|\nabla \tilde{u}(x)|) d x \leq \int_{A} \frac{1}{2 \pi} \int_{|\omega|=1} l(|\nabla u(\omega|x|)|) d \omega d x .
$$

Being the function $v(|x|)=\frac{1}{2 \pi} \int_{|\omega|=1} l(|\nabla u(\omega|x|)|)$ radial, passing to spherical coordinates we obtain

$$
\begin{aligned}
& \int_{A} l(|\nabla \tilde{u}(x)|) d x \leq 2 \pi \int_{\frac{1}{2}}^{1} v(r) r d r \\
&=\int_{\frac{1}{2}}^{1} \int_{|\omega|=1} l(|\nabla u(\omega r)|) d \omega r d r=\int_{\Omega} l(|\nabla u(x)|) d x
\end{aligned}
$$

so that $\tilde{u}$ is a radial solution to problem (3.1). This contradicts point a). 


\section{Sufficient conditions for the existence of solutions}

It is convenient to set $L(\xi)=l(|\xi|)=\sqrt{1+|\xi|^{2}}$.

The sufficiency is first of all based on the requirement that $\partial \Omega$ be smooth (further conditions on $\partial \Omega$ will be discussed later) and that the boundary condition $\phi$ be smooth; it is convenient to assume that $\phi$ is defined and twice differentiable in an open set containing $\bar{\Omega}$.

The reasoning that leads to the proof of existence of solutions is long. One looks for solutions for the minimization problem restricted to the (convex) set of functions that satisfy the given boundary and are Lipschitzian with a given Lipschitz constant. If this set is not empty, a solution to the restricted problem exists, as a function with the given Lipschitz condition. Increasing the allowed Lipschitz constant, in general the solution found changes. There is, however, a further result, based on strict convexity (the minimal area functional is strictly convex): assume that, for a certain $\Lambda$, the minimization problem restricted to the functions of Lipschitz constant $\Lambda$ admits a solution $u_{\Lambda}$, and that it happens that the Lipschitz constant of $u_{\Lambda}$ is strictly less than $\Lambda$. Then, the same $u_{\Lambda}$ is a solution to the problem considered over the linear space of all Lipschitz functions (satisfying the given boundary conditions). This reasoning boils down to the following principle, applied to $u_{\Lambda}$ : if we can "a priori" bound the Lipschitz constant of a solution, then a solution will exist.

How to a priori bound the Lipschitz constant of $u$ ? Again, the reasoning is complex. There are some "a priori" bounds for $u$; the simplest is the following: if a constant $k$ is such that $\phi(x) \leq k$ for $x \in \partial \Omega$, then $u(x) \leq k$ for $x$ in $\Omega$. A more difficult estimate is the following: if $u$ is a solution, then, for every $\varepsilon$,

$$
\begin{aligned}
\sup \left\{\frac{|u(x)-u(y)|}{|x-y|}: x \text { and } y \text { in } \bar{\Omega}\right\} & \\
=\sup \left\{\frac{|u(x)-u(y)|}{|x-y|}:\right. & \text { and } y \text { in } \bar{\Omega} ;|x-y| \leq \varepsilon\} \\
& =\sup \left\{\frac{|u(x)-u(y)|}{|x-y|}: x \in \partial \Omega, y \in \Omega\right\} .
\end{aligned}
$$

This means that it is enough to bound the Lipschitz constant of $u$ only on the boundary: if a bound is found, this will be the bound at any point $x \in \Omega$. Hence, the whole proof of the existence of a solution, among all functions that are Lipschitzian and satisfy the boundary conditions, amounts to showing that the solution $u$ is (uniformly) Lipschitzian when $x \in \partial \Omega$.

Based on the previous line of thought, the proof of the existence of a solution to the minimal area problem, then, aims at finding two things: a) a "neighborhood of the boundary of $\Omega$ ", i.e., a subset $\Omega_{\delta}$ of $\Omega, \Omega_{\delta}=\{x \in \Omega$ : 
$d(x)<\delta\}$, where by $d(x)$ we mean the distance of $x \in \Omega$ to the complement of $\Omega$, and b) a "supersolution" $v$, i.e., a twice differentiable function such that we have at once

$$
\operatorname{div} \nabla L(\nabla v(x)) \leq 0 \quad \text { a.e. in } \Omega_{\delta}
$$

and $v(x) \geq u(x)$ for $x \in \partial \Omega_{\delta}$. Since $u$ is unknown, this last requirement will be met simply by constructing $v$ such that $v(x)=\phi(x)$ for $x \in \partial \Omega$ while $u(x) \geq k$ where $k$ is such that $k \geq \phi(x)$, for any $x \in \partial \Omega$. The supersolution forces the solution to stay below, and having differential quotients $\frac{v(x)-v(y)}{|x-y|}$ bounded (since we shall build a Lipschitzian $v$ ) in particular bounds from above the differential quotients $\frac{u(x)-u(y)}{|x-y|}$ when $y \in \partial \Omega$, so that $v(y)=u(y)=\phi(y)$. To bound it from below, we need an analogous construction for a subsolution.

We wish to point out explicitly that this part of the proof is based only on the fact that $L$ depends only on $\xi$ and that $L$ as a function of $\xi$ is strictly convex.

The construction of a supersolution $v$ for $L(\xi)=\sqrt{1+|\xi|^{2}}$. The construction depends on the function $d$, distance from a point $x \in \Omega$ to $\partial \Omega$; some properties of this function are very relevant for what follows. In general, the distance from a closed set is a Lipschitzian function with Lipschitz constant one. When the gradient exists, it is a unit vector pointing from the nearest point at the boundary to $x \in \Omega$. For our purposes, the boundary of $\Omega$ is $C^{2}$, hence its curvatures are uniformly bounded; this implies the existence of $h$ such that when $d(x) \leq h$, the projection of $x$ to the boundary consists of a single point and the gradient of $d$ at $x$ exists and is $C^{1}$. So, in the remainder of the construction, we shall assume that $d \leq h$. Hence $\nabla d(x)$ exists and $|\nabla d(x)|=1$.

We set, for $x \in \Omega$,

$$
v(x)=\psi(d(x))+\phi(x)
$$

where $\psi$, to be defined, is such that $\psi(0)=0$; then, the function $v$ is such that $\left.v\right|_{\partial \Omega}=\left.u\right|_{\partial \Omega}=\left.\phi\right|_{\partial \Omega}$. Then $\nabla v(x)=\psi^{\prime}(d(x)) \nabla d(x)+\nabla \phi(x)$.

We denote by $H_{f}$ the Hessian matrix of the function $f$; in particular, from $|\nabla d(x)|^{2} \equiv 1$ we obtain $H_{d} \nabla d=0$; this property will be of use later.

For the function $v$ we have

$$
H_{v}=H_{\phi}+\psi^{\prime}(d) H_{d}+\psi^{\prime \prime}(d) \nabla d \otimes \nabla d
$$

(by $\xi \otimes \xi$ we mean the matrix $\left.\left(\xi_{i} \xi_{j}\right)_{i, j}\right)$ and

$$
|\nabla v|^{2}=|\nabla \phi|^{2}+\psi^{\prime}(d)^{2}+2\langle\nabla \phi, \nabla d\rangle .
$$


From

$$
\nabla L(\xi)=\frac{\xi}{\sqrt{1+|\xi|^{2}}}
$$

we obtain

$$
\operatorname{div} \nabla L(\nabla v)=\frac{1}{\left(1+|\nabla v|^{2}\right)^{\frac{3}{2}}}\left[\left(1+|\nabla v|^{2}\right) \Delta v-(\nabla v)^{T} H_{v} \nabla v\right] .
$$

Hence, in order to obtain $\operatorname{div} \nabla L(\nabla v) \leq 0$, it is enough to have $\left(1+|\nabla v|^{2}\right) \Delta v-$ $(\nabla v)^{T} H_{v} \nabla v \leq 0$. This is a first simplification, coming from the special form of $L$.

After a few simplifications, we obtain

$$
\begin{aligned}
(\nabla v)^{T} H_{v} \nabla v & =(\nabla \phi)^{T} H_{\phi} \nabla \phi+2 \psi^{\prime}(\nabla \phi)^{T} H_{\phi} \nabla d+\left(\psi^{\prime}\right)^{2}(\nabla d)^{T} H_{\phi} \nabla d \\
& +\psi^{\prime}(\nabla \phi)^{T} H_{d} \nabla \phi+\psi^{\prime \prime}\langle\nabla \phi, \nabla d\rangle^{2}+\psi^{\prime} \psi^{\prime \prime}\langle\nabla \phi, \nabla d\rangle+\left(\psi^{\prime}\right)^{2} \psi^{\prime \prime}
\end{aligned}
$$

and

$$
\left(1+|\nabla v|^{2}\right) \Delta v=\left(\Delta \phi+\psi^{\prime \prime}+\psi^{\prime} \Delta d\right)\left(1+|\nabla \phi|^{2}+2 \psi^{\prime}\langle\nabla d, \nabla \phi\rangle+\left(\psi^{\prime}\right)^{2}\right)
$$

Hence, in order for $v$ to be a supersolution, the following inequality has to hold:

$$
\begin{aligned}
(\Delta \phi+ & \left.\psi^{\prime \prime}+\psi^{\prime} \Delta d\right)\left(1+|\nabla \phi|^{2}+2 \psi^{\prime}\langle\nabla d, \nabla \phi\rangle+\left(\psi^{\prime}\right)^{2}\right) \\
& +(\nabla \phi)^{T} H_{\phi} \nabla \phi+2 \psi^{\prime}(\nabla \phi)^{T} H_{\phi} \nabla d+\left(\psi^{\prime}\right)^{2}(\nabla d)^{T} H_{\phi} \nabla d \\
& +\psi^{\prime}(\nabla \phi)^{T} H_{d} \nabla \phi+\psi^{\prime \prime}\langle\nabla \phi, \nabla d\rangle^{2}+\psi^{\prime} \psi^{\prime \prime}\langle\nabla \phi, \nabla d\rangle+\left(\psi^{\prime}\right)^{2} \psi^{\prime \prime} \leq 0 .
\end{aligned}
$$

The previous expression contains terms in $\psi^{\prime}, \psi^{\prime \prime}$ and mixed terms, and the way to order it is part of the problem. Again after a few simplifications, we can write the expression as

$$
\begin{gathered}
\Delta \phi+|\nabla \phi|^{2} \Delta \phi+(\nabla \phi)^{T} H_{\phi} \nabla \phi \\
+\psi^{\prime}\left[2\langle\nabla d, \nabla \phi\rangle \Delta \phi-2(\nabla \phi)^{T} H_{\phi} \nabla d-(\nabla \phi)^{T} H_{d} \nabla \phi+\left(1+\left|(\nabla \phi)^{T} H_{d} \nabla \phi\right|^{2}\right) \Delta \phi\right] \\
+\left(\psi^{\prime}\right)^{2}\left[\Delta \phi+2\langle\nabla d, \nabla \phi\rangle \Delta d-(\nabla d)^{T} H_{\phi} \nabla d\right]+\left(\psi^{\prime}\right)^{3} \Delta d \\
+\psi^{\prime \prime}\left(1+|\nabla \phi|^{2}-\langle\nabla d, \nabla \phi\rangle^{2}\right) .
\end{gathered}
$$


The function $\psi$ we are trying to build will have a positive and very large first derivative $\psi^{\prime}$; hence, the term $\left(\psi^{\prime}\right)^{3} \Delta d$ will play a fundamental role: we want this term to be non-positive. Hence, we have to discuss the sign of the function $\Delta d$.

We represent locally $\partial \Omega$ setting the origin of the coordinates at a point $x^{*}=\left(x_{1}^{*}, \ldots, x_{N}^{*}\right)$ where $e_{N}$ is the direction of the normal pointing inside the set $\Omega$. Then, as it is known, for $x$ sufficiently close to $\partial \Omega$, we can write

$$
\Delta d(x)=-\sum_{1}^{N} \frac{k_{i}}{1-k_{i} d(x)}
$$

where the $k_{i}$ are the principal curvatures of $\partial \Omega$ at the point $y(x)$ of the nearest projection of $x$ on $\partial \Omega$. An easy computation shows that $\Delta d$, seen as a function of $d$ keeping $x$ fixed, is always decreasing; hence, if $\Delta d$ computed at $d=0$ is non-positive, it will remain non-positive as we move to the interior: the condition

$$
-\sum_{1}^{N} k_{i} \leq 0
$$

at every point of the boundary (i.e., that the mean curvature is non-negative on $\partial \Omega$ ) assures that the term $\left(\psi^{\prime}\right)^{3} \Delta d \leq 0$ for $\psi^{\prime} \geq 0$. This condition on the mean curvature of the boundary is the basic requirement for the existence of solutions: we notice that this condition is not verified by the annulus $A$ in the example of non-existence. In addition, we notice that this condition on the boundary is introduced in this discussion as a sufficient condition; remarkably, it has been shown by Jenkins and Serrin [14] that it is necessary as well: whenever a (bounded and smooth) set $\Omega$ violates this condition, it is possible to build a boundary datum $\phi$ such that the corresponding minimization problem has no solutions.

Going back to the construction of $\psi$, we notice that the terms involving $\phi$ and $d$ are bounded, due to the smoothness of $\phi$ and of $\nabla \Omega$. Then we can show that $\operatorname{div} \nabla L(\nabla v) \leq 0$ if the following problem can be solved: positive constants $A, M$ and $U$ are given; find a $\delta>0$ and a twice differentiable function $\psi$ defined on $[0, \delta]$ such that:

$$
\psi(0)=0 ; \psi(\delta) \geq U, \psi^{\prime}(d) \geq M \text { and } \psi^{\prime \prime}(d) \leq 0 \text { for } d \in[0, \delta]
$$

and such that, on $[0, \delta]$,

$$
\psi^{\prime \prime}(d)+A\left(\psi^{\prime}(d)\right)^{2}=0 .
$$


A solution $\psi$ to the differential equation and satisfying $\psi(0)=0$ is given by

$$
\psi(d)=\frac{1}{A} \ln \left(\frac{d+k}{k}\right)
$$

with $k$ an arbitrary constant; we set $\frac{1}{A} \ln \left(\frac{\delta+k}{k}\right)=U$ to satisfy the condition at $\delta$, and we obtain $k=\frac{\delta}{e^{A U}-1}$, so that now the solution is completely determined; we still have to satisfy $\psi^{\prime}(d) \geq M$ for $d \in[0, \delta]$. Since $\psi^{\prime \prime}(d) \leq 0$ on $[0, \delta]$, we can as well satisfy the condition $\psi^{\prime}(\delta) \geq M$. Since

$$
\psi^{\prime}(d)=\frac{1}{A\left(d+\frac{\delta}{e^{A U}-1}\right)}
$$

we obtain

$$
\psi^{\prime}(\delta)=\frac{1}{A \delta\left(1+\frac{1}{e^{A U}-1}\right)} ;
$$

then it is enough to take $\delta$ sufficiently small to satisfy $\psi^{\prime}(\delta) \geq M$.

Hence, we have built a function $v$ that satisfies $v(x)=u(x)$ at $\partial \Omega$ and that bounds a solution $u$ from above in $\Omega_{\delta}$, so that

$$
\sup \left\{\frac{u(x)-u(y)}{|x-y|}: x \in \partial \Omega, y \in \Omega_{\delta}\right\} \leq \sup \left\{\frac{v(x)-v(y)}{|x-y|}: x \in \partial \Omega, y \in \Omega_{\delta}\right\}
$$

and the term at the right is bounded by $\psi^{\prime}(0)+\sup \{|\nabla \phi|: x \in \bar{\Omega}\}$. Once the analogous construction for a subsolution is performed, in order to bound the differential quotient from below, the proof of existence is achieved.

A note on the solution. The construction above finds a solution among those functions in $\mathbb{L}$, the linear space of Lipschitzian functions, that satisfy the boundary conditions. Are we sure that the solution found solves the problem in the larger space of those functions in $W^{1,1}(A)$ that satisfy the same boundary conditions? It is known that for variational problems, the Lavrentiev phenomenon can occur [11. Calling $I(v)$ the integral functional computed at a function $v$ satisfying the boundary conditions, it can happen that $\inf \{I(v): v \in \mathbb{L}\}<\inf \left\{I(v): v \in W^{1,1}(A)\right\}$ : if this were to happen, the solution found with the construction shown above would not be a true solution of the problem. Fortunately this phenomenon cannot happen in this case, simply because the integrand $\xi \rightarrow L(|\xi|)$ for the minimal surface problem is 
Lipschitzian of constant 1 . Then, let $\left(v_{n}\right)$ be a sequence of smooth mappings converging to $u$ in $W^{1,1}(A)$. We have:

$$
\left|\int_{A} L\left(\left|\nabla v_{n}\right|\right) d x-\int_{A} L(|\nabla u|) d x\right| \leq \int_{A}\left|\nabla v_{n}-\nabla u\right| d x \rightarrow 0
$$

from the convergence in $W^{1,1}(A)$. Hence, the phenomenon cannot occur and the solution we built is a solution among functions in $W^{1,1}(A)$.

\section{The example of a problem without existence of solutions revisited}

The phenomenon of having boundary conditions and domains of integration such that the corresponding minimization problem admits no solutions certainly is not limited to the minimal area functional. For instance, what happens if, instead of considering $l(t)=\sqrt{1+t^{2}}$, we consider $l(t)=\left(1+t^{4}\right)^{\frac{1}{4}}$ ?

From now on, $L(\xi)=l(|\xi|)$ does not denote the minimal surface integrand anymore, but a generic strictly convex smooth function of slow growth; more precisely we shall assume that $l$ has a minimum equal to zero at $t=0$ (hence, in the case of the minimal area functional, we should consider $l(t)=\sqrt{1+t^{2}}-$ 1). We shall need the polar or Legendre transform of $l$ [15], defined by $l^{*}(p)=\sup _{t}(p t)-l(t)$. Under our conditions on $l, l^{*}(p) \geq 0$ and $l^{*}$ is a (possibly extended-valued) convex function, whose effective domain is denoted by $\operatorname{Dom}\left(l^{*}\right)$. Further properties will be discussed later.

Consider the problem of minimizing

$$
\int_{A} l(|\nabla u(x)|) d x
$$

with the same boundary condition as for 3.1), i.e.,

$$
u(x)=0 \text { for }|x|=1 ; \quad u(x)=-\alpha \text { for }|x|=\frac{1}{2}
$$

and, as for problem (3.1), let us look for radial solutions. In addition, we assume $-\alpha \geq 1$.

Assume that we have a radial solution $\tilde{u}$; then, the Euler-Lagrange equation implies that

$$
\frac{d}{d r}\left(l^{\prime}\left(\tilde{u}^{\prime}(r)\right) r\right)=0
$$


i.e., that

$$
l^{\prime}\left(\tilde{u}^{\prime}(r)\right)=l^{\prime}\left(\tilde{u}^{\prime}\left(\frac{1}{2}\right)\right)\left(\frac{1}{2 r}\right) .
$$

Since $\tilde{u}(1)-\tilde{u}\left(\frac{1}{2}\right)=-\alpha$, for some $\hat{r} \in\left(\frac{1}{2}, 1\right)$ we must have $\tilde{u}^{\prime}(\hat{r}) \geq 2(-\alpha)$; since the right hand side of $(3.3)$ is non-increasing and $l^{\prime}$ is non-decreasing, we infer that $l^{\prime}\left(\tilde{u}^{\prime}\left(\frac{1}{2}\right)\right) \geq l^{\prime}\left(\tilde{u}^{\prime}(\hat{r})\right) \geq l^{\prime}(-2 \alpha) \geq l^{\prime}(1)$.

Solve 3.3 as $\tilde{u}^{\prime}(r)=\left(l^{*}\right)^{\prime}\left(l^{\prime}\left(\tilde{u}^{\prime}\left(\frac{1}{2}\right)\right)\left(\frac{1}{2 r}\right)\right)$. Since $r \rightarrow\left(\frac{1}{2 r}\right)$ is convex for positive $r$, on the interval $\frac{1}{2} \leq r \leq 1$ we have $\left(\frac{1}{2 r}\right) \leq-\left(r-\frac{1}{2}\right)+1=\frac{3}{2}-r$ and, since $\left(l^{*}\right)^{\prime}$ is non-decreasing, $\left(l^{*}\right)^{\prime}\left(l^{\prime}\left(\tilde{u}^{\prime}\left(\frac{1}{2}\right)\right)\left(\frac{1}{2 r}\right)\right) \leq\left(l^{*}\right)^{\prime}\left(l^{\prime}\left(\tilde{u}^{\prime}\left(\frac{1}{2}\right)\right)\left(\frac{3}{2}-r\right)\right)$. Hence

$$
\begin{aligned}
\int_{\frac{1}{2}}^{1} \tilde{u}^{\prime}(r) d r & =\int_{\frac{1}{2}}^{1}\left(l^{*}\right)^{\prime}\left(l^{\prime}\left(\tilde{u}^{\prime}\left(\frac{1}{2}\right)\right)\left(\frac{1}{2 r}\right)\right) d r \\
& \leq \int_{\frac{1}{2}}^{1}\left(l^{*}\right)^{\prime}\left(l^{\prime}\left(\tilde{u}^{\prime}\left(\frac{1}{2}\right)\right)\left(\frac{3}{2}-r\right)\right) d r \\
& =\frac{1}{l^{\prime}\left(\tilde{u}^{\prime}\left(\frac{1}{2}\right)\right)} \int_{y(1)}^{y\left(\frac{1}{2}\right)}\left(l^{*}\right)^{\prime}(y) d y=\frac{1}{l^{\prime}\left(\tilde{u}^{\prime}\left(\frac{1}{2}\right)\right)}\left[l^{*}\left(y\left(\frac{1}{2}\right)\right)-l^{*}(y(1))\right] .
\end{aligned}
$$

Hence,

$$
\left|\int_{\frac{1}{2}}^{1} \tilde{u}^{\prime}(r) d r\right| \leq \frac{1}{l^{\prime}(1)} M
$$

where $M=\sup \left\{l^{*}(p): p \in \operatorname{Dom}\left(l^{*}\right)\right\}$.

Then, whenever $M$ is finite and $|\alpha|$ is strictly larger than $\frac{1}{l^{\prime}(1)} M$, problem (3.2) admits no radial solution.

For the minimal area functional, $l(t)=\sqrt{1+t^{2}}-1$, we obtain that, for $p \in[-1,1]$,

$$
l^{*}(p)=1-\sqrt{1-p^{2}}
$$

while $l^{*}(p)=+\infty$ for $p \notin[-1,1]$; hence, $M=1$. A similar computation for $f(t)=\left(1+t^{4}\right)^{\frac{1}{4}}-1$ gives $l^{*}(p)=-\left(1-p^{\frac{4}{3}}\right)^{\frac{3}{4}}+1$ for $|p| \leq 1, l^{*}(p)=+\infty$ otherwise; $M$ is again $=1$.

We have found a different way for showing the existence of a boundary condition on $A$ such that the corresponding minimization problem for the minimal area functional admits no solutions. Moreover, this property of nonexistence of solutions is not restricted to the minimal area functional, but it is common to all functionals such that the image of $l^{*}$ is bounded. 
A condition involving the polar. The polar (or Legendre transform) of a function $L$, defined by setting

$$
L^{*}(p)=\sup _{\xi \in \mathbb{R}^{N}}[\langle p, \xi\rangle-L(\xi)]
$$

is a convex, extended-valued function. We are interested in Lagrangians depending on the norm of $\nabla v$, hence our $l$ is defined on $\mathbb{R}$ or, possibly on a convex subset of it. We wish to examine the implications of the following condition.

Assumption 1. The polar $l^{*}$ is a continuous extended-valued function on $\mathbb{R}$.

When $l^{*}$ is defined over the whole $\mathbb{R}$ (with finite values), it is continuous by convexity; otherwise, the effective domain of $l^{*}$ is an interval; the condition of continuity implies that this interval must be open. When $l$ is of superlinear growth, we can prove that its polar is defined over $\mathbb{R}$, hence Assumption 1 is always satisfied by mappings $l$ of superlinear growth. The functional of minimal area has a polar whose effective domain is the closed interval $[-1,1]$, so that Assumption 1 is not verified.

Let us consider a different map of linear growth. The map

$$
l(t)= \begin{cases}|t|-\sqrt{|t|}+\frac{1}{8} \alpha+\frac{1}{2} \beta+\gamma & \text { for }|t| \geq \frac{1}{2}, \\ \frac{1}{4} \alpha|t|^{4}+\frac{1}{2} \beta|t|^{2} & \text { for }|t| \leq \frac{1}{2},\end{cases}
$$

where $\alpha=\frac{3}{\sqrt{2}}-2, \beta=\frac{3+\sqrt{2}}{2}-\frac{9}{4 \sqrt{2}}$ and $\gamma=\sqrt{2}-1$, is strictly convex, $C^{2}$ and of linear growth; again, Assumption 1 is satisfied. In fact, for this $l$ we have (we compute $l^{*}$ only for $|p| \geq 1-\frac{1}{\sqrt{2}}$ ),

$$
l^{*}(p)= \begin{cases}\frac{1}{4} \frac{1}{1-|p|} & \text { for } 1>|p| \geq 1-\frac{1}{\sqrt{2}}, \\ +\infty & \text { for }|p| \geq 1\end{cases}
$$

so that $l^{*}$ is continuous as a map to $\mathbb{R} \cup\{+\infty\}$. Hence, this function provides an example of a map of linear growth such that Assumption 1 holds.

Assumption 1, in a slightly different form, was introduced in [10] for a problem of slow growth on the parametrizations of a given curve; in [3], it was shown that Assumption 1 implies that solutions to an autonomous functional defined over a one-dimensional set are Lipschitzian; in [7] it is shown that under Assumption 1, an autonomous functional as before actually admits solutions, through a variant of the Direct method; in fact, no coercivity is assumed; it is proved that, when Assumption 1 is satisfied, from a minimizing 
sequence we can select a subsequence such that suitable reparametrizations of the subsequence converge and still form a minimizing sequence. Finally, the problem on a multi-dimensional space was met in [9]. To state the main result of [9], we need some notations.

The class $\mathbb{L}$ of Lagrangians $L$ we shall consider is

$$
\begin{gathered}
\mathbb{L}=\left\{L(\xi)=l(|\xi|): l: \mathbb{R} \rightarrow \mathbb{R}^{+} \text {is strictly convex, } l(t)=l(-t),\right. \\
\left.\quad l \in C^{2} \text { and } l^{\prime \prime} \text { is non-increasing }\right\}
\end{gathered}
$$

The class considered contains functionals of slow growth; it contains $l(t)=\frac{1}{2} t^{2}$ and functionals of slower growth.

To describe the class of boundary conditions and of sets $\Omega$ that are allowed, set

$$
\mathbb{F}=\left\{\phi \in C^{2}(\bar{\Omega})\right\} \quad \text { and } \quad \mathbb{O}=\left\{\Omega \text { open and bounded: } \partial \Omega \in C^{2}\right\} .
$$

Hence, the paper deals with smooth boundary conditions and smooth sets $\Omega$.

In [9], the following theorem was proved.

Theorem 3.2. Let $L \in \mathbb{L}$. Then, for every $\phi \in \mathbb{F}$, for every $\Omega \in \mathbb{O}$, problem (3.1) admits a (unique) solution $\tilde{u} \in W^{1,1}(\Omega)$ if and only if Assumption 1 holds.

For the non-parametric minimal area problem Assumption 1 is not satisfied and it is not true that for every $\phi \in \mathbb{F}$, for every $\Omega \in \mathbb{O}$, problem (3.1) admits a solution.

The necessity of Assumption 1 was essentially proved in the discussion of the previous section. Here we wish to discuss the sufficiency part.

\section{Sufficiency under Assumption 1}

We shall denote the domain of $l^{*}$ by $\left(-p^{*}, p^{*}\right)$, with $p^{*}$ possibly $+\infty$. To show that Assumption 1 is sufficient for the existence of a solution, no matter what the boundary condition is in the set $\mathbb{F}$, the argument is the same as in the proof of existence for the minimal area problem. In fact, the preliminary discussion for that proof was based only on the property that $l$ is strictly convex, a condition retained in the new setting. Hence, the proof is based on the construction of a supersolution (and, clearly, of a subsolution as well). In the case of the minimal area problem, the possibility of defining a supersolution was based on the discussion of the sign of the Laplacian of the distance function, that was in itself based on a condition on the mean curvature of $\partial \Omega$. 
No such a condition exists in the statement of the present theorem, hence the construction of a supersolution will be based on substantially different arguments.

We shall build the supersolution $v$ as it was done in the case of the minimal area problem: we set again, for $x \in \Omega$,

$$
v(x)=\psi(d(x))+\phi(x)
$$

where $\psi$, to be defined, is such that $\psi(0)=0$; then, the function $v$ is such that $\left.v\right|_{\partial \Omega}=\left.u\right|_{\partial \Omega}=\left.\phi\right|_{\partial \Omega}$ and $\nabla v(x)=\psi^{\prime}(d(x)) \nabla d(x)+\nabla \phi(x)$. We obtain

$$
\begin{aligned}
& \operatorname{div} \nabla L(\nabla u(x))=\frac{l^{\prime}}{|\nabla u|}\left(\psi^{\prime} \Delta d+\psi^{\prime \prime}+\Delta \phi\right) \\
& +\frac{\left(l^{\prime \prime}-\frac{l^{\prime}}{|\nabla u|}\right)}{|\nabla u|^{2}}\left(\psi^{\prime} \nabla d+\nabla \phi\right)^{T}\left(\psi^{\prime \prime} \nabla d \otimes \nabla d+\psi^{\prime} H_{d}+H_{\phi}\right)\left(\psi^{\prime} \nabla d+\nabla \phi\right) .
\end{aligned}
$$

We still have to find $\psi$ that makes this expression negative. Notice that in the corresponding expression for $l(t)=\sqrt{1+t^{2}}$, we were able to find an expression for $\operatorname{div} \nabla L(\nabla u)$ that was explicit in $\psi^{\prime}$ and its powers, so that we could arrange its terms according to the different powers of $\psi^{\prime}$; here, $\psi^{\prime}$ appears also hidden in the non-linear expressions $l^{\prime}$ and $l^{\prime \prime}$, besides being in $\nabla u$. Expanding the products we obtain

$$
\begin{array}{r}
\operatorname{div} \nabla L(\nabla u(x))=\frac{\left(\psi^{\prime}\right)^{2}}{|\nabla u|^{2}} \psi^{\prime \prime}\left(l^{\prime \prime}-\frac{l^{\prime}}{|\nabla u|}\right)+\frac{\psi^{\prime \prime}}{|\nabla u|^{2}}\left(\langle\nabla \phi, \nabla d\rangle^{2}\right. \\
\left.+2 \psi^{\prime}\langle\nabla \phi, \nabla d\rangle\right)\left(l^{\prime \prime}-\frac{l^{\prime}}{|\nabla u|}\right)+\psi^{\prime \prime} \frac{l^{\prime}}{|\nabla u|} \frac{\left(\psi^{\prime}\right)^{2}}{|\nabla u|^{2}} \frac{|\nabla u|^{2}}{\left(\psi^{\prime}\right)^{2}}+\frac{l^{\prime}\left[\psi^{\prime} \Delta d+\Delta \phi\right]}{|\nabla u|} \\
+\frac{\left(l^{\prime \prime}-\frac{l^{\prime}}{|\nabla u|}\right)}{|\nabla u|^{2}}\left(\psi^{\prime} \nabla \phi^{T} H_{d} \nabla \phi+(\nabla \phi)^{T} H_{\phi} \nabla \phi+2 \psi^{\prime}(\nabla \phi)^{T} H_{\phi} \nabla d\right. \\
\left.+\left(\psi^{\prime}\right)^{2}(\nabla d)^{T} H_{\phi} \nabla d\right) .
\end{array}
$$

After a few estimates we obtain that, in order to have $\operatorname{div} \nabla L(\nabla u(x)) \leq 0$, it is enough to have

$$
0 \geq \psi^{\prime \prime} l^{\prime \prime}\left\{1+\frac{1}{\left(\psi^{\prime}\right)^{2}}\left(\langle\nabla \phi, \nabla d\rangle^{2}+2 \psi^{\prime}\langle\nabla \phi, \nabla d\rangle\right)\right\}
$$




$$
\begin{gathered}
+l^{\prime}\left\{\frac{|\nabla u| \Delta d}{\psi^{\prime}}+\frac{|\nabla u| \Delta \phi}{\left(\psi^{\prime}\right)^{2}}-\frac{1}{\psi^{\prime}|\nabla u|}(\nabla \phi)^{T} H_{d} \nabla \phi-\frac{1}{\left(\psi^{\prime}\right)^{2}|\nabla u|}(\nabla \phi)^{T} H_{\phi} \nabla \phi\right. \\
\left.-\frac{2}{\psi^{\prime}|\nabla u|}(\nabla d)^{T} H_{\phi} \nabla \phi-\frac{1}{|\nabla u|}(\nabla d)^{T} H_{\phi} \nabla d\right\} \\
+l^{\prime \prime}\left(\frac{1}{\psi^{\prime}} \nabla \phi^{T} H_{d} \nabla \phi+\frac{1}{\left(\psi^{\prime}\right)^{2}}(\nabla \phi)^{T} H_{\phi} \nabla \phi+\frac{2}{\psi^{\prime}}(\nabla d)^{T} H_{\phi} \nabla \phi+(\nabla d)^{T} H_{\phi} \nabla d\right) \\
=\psi^{\prime \prime} l^{\prime \prime}\left\{1+\frac{1}{\left(\psi^{\prime}\right)^{2}}\left(\langle\nabla \phi, \nabla d\rangle^{2}+2 \psi^{\prime}\langle\nabla \phi, \nabla d\rangle\right)\right\}+l^{\prime} A+l^{\prime \prime} B,
\end{gathered}
$$

where

$$
\begin{aligned}
A= & \frac{|\nabla u| \Delta d}{\psi^{\prime}}+\frac{|\nabla u| \Delta \phi}{\left(\psi^{\prime}\right)^{2}}-\frac{1}{\psi^{\prime}|\nabla u|}(\nabla \phi)^{T} H_{d} \nabla \phi-\frac{1}{\left(\psi^{\prime}\right)^{2}|\nabla u|}(\nabla \phi)^{T} H_{\phi} \nabla \phi \\
& -\frac{2}{\psi^{\prime}|\nabla u|}(\nabla d)^{T} H_{\phi} \nabla \phi-\frac{1}{|\nabla u|}(\nabla d)^{T} H_{\phi} \nabla d
\end{aligned}
$$

and

$$
B=\frac{1}{\psi^{\prime}} \nabla \phi^{T} H_{d} \nabla \phi+\frac{1}{\left(\psi^{\prime}\right)^{2}}(\nabla \phi)^{T} H_{\phi} \nabla \phi+\frac{2}{\psi^{\prime}}(\nabla d)^{T} H_{\phi} \nabla \phi+(\nabla d)^{T} H_{\phi} \nabla d .
$$

Then, taking $\psi^{\prime}$ sufficiently large, it is enough to have

$$
\frac{1}{2} \psi^{\prime \prime} l^{\prime \prime}+l^{\prime} A+l^{\prime \prime} B \leq 0 .
$$

Consider $A$; a constant $h_{\Delta}$ exists such that, for $\psi^{\prime}$ large, $A \leq 2 h_{\Delta}$ (no assumptions on the sign of $\Delta d$ !). Similarly, for $\psi^{\prime} \geq P$ we have $B \leq 2 h_{\phi}$; hence, to have $\operatorname{div} \nabla L(\nabla u(x)) \leq 0$, it is enough to have

$$
\psi^{\prime} \geq P \text { and } \frac{1}{2} \psi^{\prime \prime} l^{\prime \prime}(|\nabla u|)+l^{\prime}(|\nabla u|) 2 h_{\Delta}+l^{\prime \prime}(|\nabla u|) 2 h_{\phi} \leq 0 .
$$

In order to simplify this expression, we notice that the assumption of slow growth implies that there exist $\tau$ and $\tilde{\lambda}: t \geq \tau$ implies

$$
l^{\prime \prime}(t) \leq \tilde{\lambda} l^{\prime}(t)
$$

Then to have (3.4) it is enough to solve

$$
\psi^{\prime \prime}(d(x)) l^{\prime \prime}(|\nabla u(x)|)+C l^{\prime}(|\nabla u(x)|) \leq 0 .
$$


The above can be seen as a differential inequality for $v(d)=\psi^{\prime}(d): \psi^{\prime}$ enters, through $\nabla u$, in the argument of $l^{\prime}$ and $l^{\prime \prime}$. For large values of $\psi^{\prime}$, we have $|\nabla u| \sim \psi^{\prime}$; then, we consider a solution $v$ to the simplified differential equation

$$
\frac{l^{\prime \prime}(2 v(d))}{l^{\prime}(2 v(d))} 2 v^{\prime}(d)=-2 C
$$

that gives

$$
l^{\prime}(2 v(d))=l^{\prime}(2 v(0)) e^{-2 C d}
$$

and further approximates it; we show that $\tilde{v}$, a solution to

$$
l^{\prime}(2 \tilde{v}(d))=l^{\prime}(2 v(0))(1-2 C d),
$$

is such that

$$
\tilde{v}(d) \leq v(d)
$$

To show that $\delta>0$ can be found so that $v(\delta) \geq \tilde{P}$ and such that the condition

$$
u(x)=\psi(d(x))+\phi(x) \geq U
$$

is satisfied for $x$ such that $d(x)=\delta$, it is enough to require that

$$
\int_{0}^{\delta} \psi^{\prime}(t) d t=\int_{0}^{\delta} v(t) d t \geq 2 U
$$

hence

$$
\int_{0}^{\delta} \tilde{v}(t) d t \geq 2 \phi \text { and } \tilde{v}(\delta) \geq \tilde{P}
$$

In (3.5), $\tilde{v}$ is given in an implicit form; solve it setting

$$
\tilde{v}(t)=\frac{1}{2}\left(l^{*}\right)^{\prime}\left(l^{\prime}(2 v(0))(1-2 C t)\right) .
$$

Set $p^{0}=l^{\prime}(2 v(0))$; the relation between $\tilde{v}(\delta)$ and $p^{0}$ is given by

$$
\tilde{v}(\delta)=\frac{1}{2}\left(l^{*}\right)^{\prime}\left(\left(l^{*}\right)^{-1}\left(\frac{1}{2} l^{*}\left(p^{0}\right)\right)\right) \quad \text { and that } \quad \int_{0}^{\delta\left(p^{0}\right)} \tilde{v}(t) d t=\frac{1}{p^{0} 8 C} l^{*}\left(p^{0}\right) .
$$


Hence, to satisfy the condition that $\psi \geq U$ when $d(x)=\delta$, it is enough to show that (recalling that the domain of $l^{*}$ is $\left(-p^{*}, p^{*}\right)$ ) we can choose $p^{0}$ sufficiently close to $p^{*}$ so as to have at once $\frac{1}{p^{0} 8 C} l^{*}\left(p^{0}\right) \geq 2 U$ and $\tilde{v}\left(\delta\left(p^{0}\right)\right) \geq \tilde{P}$.

This last fact comes from a small lemma on the properties of $l^{*}$ under Assumption 1.

\section{An introduction to regularity}

The problem. We are interested in the regularity properties of solutions to the problem of minimizing a functional of the kind

$$
I(u)=\int_{\Omega}[L(|\nabla u(x)|)+f(x) u(x)] d x
$$

with prescribed boundary conditions. (We might have more general functionals, e.g. replacing $f(x) u$ by $g(x, u)$.) The map $L$ is both differentiable and of polynomial growth, so that a solution satisfies the Euler-Lagrange equation, i.e., for every smooth $\eta$ with compact support in $\Omega$, we have

$$
\int_{\Omega}[\langle\nabla L(\nabla u(x)), \nabla \eta(x)\rangle+f(x) \eta(x)] d x=0 .
$$

We minimize an integral functional over a subset of the space of functions having (weak) first order derivatives. The solution $\tilde{u}$ simply gives a value to the integral that is the smallest among the values computed along the competing functions.

A strange phenomenon might appear: under certain conditions, the solution $\tilde{u}$, instead of having only first order derivatives, has, in addition, second order derivatives.

\section{The case of one dimensional integration set}

Assume we are minimizing the integral

$$
\int_{\alpha}^{\beta}\left[\frac{1}{2}\left|y^{\prime}(t)\right|^{2}+f(t) y(t)\right] d t, y(\alpha)=a, y(\beta)=b,
$$

where $f \in L^{2}((\alpha, \beta))$, among the functions that are in $W^{1,2}((\alpha, \beta))$ with the given boundary conditions. 
A unique solution to the problem exists, and it gives the least possible value to the integral among all the functions that have a first order derivative in $L^{2}$ and that satisfy the boundary conditions. We wish to show the surprising fact that the solution is more regular than the competing functions: it admits a second order derivative that is in $L^{2}$.

Let $x$ be the solution. First, we notice that we can obtain an a-priori estimate on $\int_{\alpha}^{\beta}\left|x^{\prime}(t)\right|^{2} d t$. In fact, the function $\left(a+\frac{b-a}{\beta-\alpha}(t-\alpha)\right)$ satisfies the given boundary conditions and clearly gives a finite value $V$ to the integral, so that $\int_{\alpha}^{\beta}\left[\frac{1}{2}\left|x^{\prime}(t)\right|^{2}+f(t) x(t)\right] d t \leq V$. Moreover, the function $y(t)=x(t)-$ $\left(a+\frac{b-a}{\beta-\alpha}(t-\alpha)\right)$ is zero at the boundary and is absolutely continuous, so that we obtain

$$
\int_{\alpha}^{\beta}|y(t)|^{2} d t \leq \frac{1}{2}(\beta-\alpha)^{2} \int_{\alpha}^{\beta}\left|y^{\prime}(t)\right|^{2} d t
$$

then,

$$
\begin{gathered}
\int_{\alpha}^{\beta}|x(t)|^{2} d t=\int_{\alpha}^{\beta}\left|a+\frac{b-a}{\beta-\alpha}(t-\alpha)+x(t)-\left(a+\frac{b-a}{\beta-\alpha}(t-\alpha)\right)\right|^{2} d t \\
\quad \leq \int_{\alpha}^{\beta} 2\left|a+\frac{b-a}{\beta-\alpha}(t-\alpha)\right|^{2} d t+(\beta-\alpha)^{2} \int_{\alpha}^{\beta}\left(x^{\prime}(t)-\frac{b-a}{\beta-\alpha}\right)^{2} d t \\
\leq \int_{\alpha}^{\beta} 2\left|a+\frac{b-a}{\beta-\alpha}(t-\alpha)\right|^{2} d t+(\beta-\alpha)^{2} \int_{\alpha}^{\beta}\left(2\left|x^{\prime}(t)\right|^{2}+2\left|\frac{b-a}{\beta-\alpha}\right|^{2}\right) d t .
\end{gathered}
$$

Hence, there are two constants $C_{0}$ and $C_{1}$, depending on the data of the problem (but not on the function $x$ ), such that

$$
\int_{\alpha}^{\beta}|x(t)|^{2} d t \leq C_{0}+C_{1} \int_{\alpha}^{\beta}\left|x^{\prime}(t)\right|^{2} d t .
$$

Hence, from $|f(t) x(t)| \leq \lambda|f(t)|^{2}+\frac{1}{\lambda}|x(t)|^{2}$, choosing $\lambda=4 C_{1}$, we obtain

$$
\begin{aligned}
V \geq \int_{\alpha}^{\beta}\left[\frac{1}{2}\left|x^{\prime}(t)\right|^{2}+f(t)\right. & x(t)] d t \geq \int_{\alpha}^{\beta}\left[\frac{1}{2}\left|x^{\prime}(t)\right|^{2}-|f(t) x(t)|\right] d t \\
& \geq \frac{1}{4} \int_{\alpha}^{\beta}\left|x^{\prime}(t)\right|^{2}-\frac{C_{0}}{4 C_{1}}(\beta-\alpha)-4 C_{1} \int_{\alpha}^{\beta}|f(t)|^{2} d t
\end{aligned}
$$


so that

$$
\int_{\alpha}^{\beta}\left|x^{\prime}(t)\right|^{2} d t \leq 4 V+4\left(\frac{C_{0}}{4 C_{1}}(\beta-\alpha)+4 C_{1} \int_{\alpha}^{\beta}|f(t)|^{2} d t\right)=K
$$

We have obtained an a priori estimate for $\int_{\alpha}^{\beta}\left|x^{\prime}(t)\right|^{2} d t$, depending on the data of the problem through the constants $V, C_{0}, C_{1}$ and on $\|f\|_{L^{2}}^{2}$.

The reasoning that leads to the proof of the additional regularity of the solution is exceedingly simple. Begin by assuming that we already knew that a second derivative exists, and try to learn something about its properties. The Euler-Lagrange equation gives

$$
\int_{\alpha}^{\beta}\left[x^{\prime}(t) \eta^{\prime}(t)+f(t) \eta(t)\right] d t=0
$$

for every variation $\eta$ that is zero at the boundary and that it is sufficiently regular. Assume that $\eta$ is itself $\phi^{\prime}$, where both $\phi$ and $\phi^{\prime}$ are zero at the boundary; then, we obtain

$$
\int_{\alpha}^{\beta}\left[x^{\prime}(t) \phi^{\prime \prime}(t)+f(t) \phi^{\prime}(t)\right] d t=0
$$

an integration by parts gives then

$$
\int_{\alpha}^{\beta} x^{\prime \prime}(t) \phi^{\prime}(t) d t=\int_{\alpha}^{\beta} f(t) \phi^{\prime}(t) d t
$$

Fix a point $t^{0} \in(\alpha, \beta)$ and let $\delta>0$ be such that $\left[t^{0}-2 \delta, t^{0}+2 \delta\right] \subset(\alpha, \beta)$ and let a variation $\eta$ be twice differentiable, such that $\eta(t) \equiv 1$ on $\left(t^{0}-\delta, t^{0}+\delta\right)$, $\eta(t) \equiv 0$ on $(\alpha, \beta) \backslash\left[t^{0}-2 \delta, t^{0}+2 \delta\right]$ and $0 \leq \eta(t) \leq 1$ everywhere. For $\phi$ take $\phi(t)=\eta^{2}(t) x^{\prime}(t)$; we have $\phi^{\prime}(t)=2 \eta(t) \eta^{\prime}(t) x^{\prime}(t)+\eta^{2}(t) x^{\prime \prime}(t)$ and the assumptions on $x$ and $\eta$ imply that $\phi \in C^{2}$, so that equation 4.2 applies; we have

$$
\int_{\alpha}^{\beta} x^{\prime \prime}(t)\left[2 \eta(t) \eta^{\prime}(t) x^{\prime}(t)+\eta^{2}(t) x^{\prime \prime}(t)\right] d t=\int_{\alpha}^{\beta} f(t) \phi^{\prime}(t) d t
$$

i.e.,

$$
\int_{\alpha}^{\beta} \eta^{2}(t)\left(x^{\prime \prime}(t)\right)^{2} d t=-\int_{\alpha}^{\beta} x^{\prime \prime}(t) 2 \eta(t) \eta^{\prime}(t) x^{\prime}(t) d t+\int_{\alpha}^{\beta} f(t) \phi^{\prime}(t) d t .
$$


This equality is the basis of the proof of regularity. At the left we have a positive integrand; hence, we have the natural inequality

$$
\int_{\alpha}^{\beta} \eta^{2}(t)\left(x^{\prime \prime}(t)\right)^{2} d t \leq \int_{\alpha}^{\beta}\left|x^{\prime \prime}(t) 2 \eta(t) \eta^{\prime}(t) x^{\prime}(t)\right| d t+\int_{\alpha}^{\beta}\left|f(t) \phi^{\prime}(t)\right| d t .
$$

For any pair $y, z$ we have $2 z y=2 z \lambda \frac{1}{\lambda} y \leq(z \lambda)^{2}+\left(\frac{1}{\lambda} y\right)^{2}$; let $z=\left|x^{\prime \prime}(t) \eta(t)\right|$, $y=\left|\eta^{\prime}(t) x^{\prime}(t)\right|$ and $\lambda=\frac{1}{2}$, to obtain

$$
\int_{\alpha}^{\beta}\left|x^{\prime \prime}(t) 2 \eta(t) \eta^{\prime}(t) x^{\prime}(t)\right| d t \leq \frac{1}{4} \int_{\alpha}^{\beta}\left|x^{\prime \prime}(t) \eta(t)\right|^{2} d t+4 \int_{\alpha}^{\beta}\left|\eta^{\prime}(t) x^{\prime}(t)\right|^{2} d t,
$$

so that 4.1 gives

$$
\frac{3}{4} \int_{\alpha}^{\beta}\left|x^{\prime \prime}(t) \eta(t)\right|^{2} d t \leq 4 \int_{\alpha}^{\beta}\left|\eta^{\prime}(t) x^{\prime}(t)\right|^{2} d t+\int_{\alpha}^{\beta}\left|f(t) \phi^{\prime}(t)\right| d t .
$$

Moreover, also

$$
\begin{aligned}
\int_{\alpha}^{\beta}\left|f(t) \phi^{\prime}(t)\right| d t \leq \int_{\alpha}^{\beta}\left[\left|\eta^{\prime}(t)\right|^{2}|f(t)|^{2}\right. & \left.+\eta(t)^{2}\left|x^{\prime}(t)\right|^{2}\right] d t \\
& +\int_{\alpha}^{\beta}\left[4|f(t)|^{2} \eta(t)^{2}+\frac{1}{4} \eta(t)^{2} x^{\prime \prime}(t)^{2}\right] d t
\end{aligned}
$$

and we have

$\int_{\alpha}^{\beta}\left|x^{\prime \prime}(t) \eta(t)\right|^{2} d t \leq \int_{\alpha}^{\beta}\left[\left(8\left|\eta^{\prime}(t)\right|+2 \eta(t)^{2}\right)\left|x^{\prime}(t)\right|^{2}+\left(8 \eta(t)^{2}+2 \eta^{\prime}(t)^{2}\right)|f(t)|^{2}\right] d t$.

From (4.1) we have a constant $H$, depending on the data of the problem and on $\eta$, such that

$$
\int_{\alpha}^{\beta}\left|x^{\prime \prime}(t) \eta(t)\right|^{2} d t \leq H
$$

In particular, on $\left[t^{0}-\delta, t^{0}+\delta\right]$ we have $\eta \equiv 1$ :

$$
\int_{t^{0}-\delta}^{t^{0}+\delta}\left|x^{\prime \prime}(t)\right|^{2} d t \leq H .
$$

We have obtained a local bound on $\int\left|x^{\prime \prime}(t)\right|^{2} d t$, depending on the data of the problem and on $t^{0}$. 
For the true problem, the existence of $x^{\prime \prime}$ is what we want to prove. The idea is to obtain bounds similar to (4.3) not on $x^{\prime \prime}$ but on the difference quotient of $x^{\prime}$ and use this information to prove the existence of $x^{\prime \prime}$.

\section{Dimension $N$}

The key to the proof of regularity lies on the following remarkable property of Sobolev functions.

Lemma 4.1. a) Let $u \in L^{2}(\Omega)$. Assume that there exists a constant $C$ such that, for every $\omega \subset \subset \Omega$ and for every $h$ sufficiently small

$$
\left\|\frac{u\left(x+h e_{s}\right)-u(x)}{h}\right\|_{L^{2}(\omega)} \leq C .
$$

Then, there exists the weak derivative $u_{x_{s}}$ and $u_{x_{s}} \in L^{2}(\Omega)$.

b) Conversely, let $u$ be in $W^{1,2}(\Omega)$. Then, there exists a constant $C$ such that, for every $\omega \subset \subset \Omega$ and for every $h$ sufficiently small

$$
\left\|\frac{u\left(x+h e_{s}\right)-u(x)}{h}\right\|_{L^{2}(\omega)} \leq C .
$$

The Laplacian. The model case is the minimization of

$$
\int_{\Omega}\left[\frac{1}{2}|\nabla u(x)|^{2}+f(x) u(x)\right] d x
$$

where the solution satisfies the Euler-Lagrange equation

$$
\int_{\Omega}[\langle\nabla \tilde{u}(x), \nabla \eta(x)\rangle+f(x) \eta(x)] d x=0
$$

for every $\eta \in W_{0}^{1,2}(\Omega)$.

In this case, $f \in L^{2}$ implies that $\tilde{u} \in W_{\text {loc }}^{2,2}(\Omega)$. Assume that we are minimizing

$$
\int_{\Omega}[L(\nabla u(x))+f(x) u(x)] d x .
$$

The conditions to be considered are

1) $L$ should be smooth,

2) $L$ has to satisfy a suitable growth condition and

3) $L$ has to be strictly convex, in a way to be discussed. 
About the requirement of smoothness, notice that $L$ need not be twice differentiable. Results allowing discontinuity of $\nabla L$ are presented in [13] and [6]. In this case the strict convexity condition becomes: there exists $m>0$ such that

$$
\langle\nabla L(x)-\nabla L(y), x-y\rangle \geq m|x-u|^{2} .
$$

A condition we have to fulfill is the condition of strict ellipticity on a matrix $H_{L}$, that requires the existence of constants $\lambda>0$ and $\Lambda$ such that for every $\xi$, for every $v$,

$$
\lambda|v|^{2} \leq v^{T} H_{L}(\xi) v \leq \Lambda|v|^{2} .
$$

For the case $L(\xi)=l(|\xi|)$, the eigenvalues of $H_{L}(\xi)$ are $\frac{l^{\prime}(|\xi|)}{|\xi|}$ and $l^{\prime \prime}(|\xi|)$ and we can write

$$
\min \left\{\frac{l(|\xi|)}{|\xi|}, l^{\prime \prime}(|\xi|)\right\}|v|^{2} \leq v^{T} H_{L}(\xi) v \leq \max \left\{\frac{l^{\prime}(|\xi|)}{|\xi|}, l^{\prime \prime}(|\xi|)\right\}|v|^{2} .
$$

Consider $l(t)=\frac{1}{p}|t|^{p}$; when $p=2$, the strict ellipticity condition is verified with $\lambda=\Lambda=1$; when $p \neq 2$, this condition is never verified: when $1<p<2$, $|t|^{p-2}$ diverges as $|t| \rightarrow 0$, and we cannot find $\Lambda$; when $p>2,|t|^{p-2} \rightarrow 0$ as $|t| \rightarrow 0$, and we cannot find $\lambda>0$. For the functional of the non-parametric minimum area problem, i.e., for $l(t)=\sqrt{1+t^{2}}$, we obtain that $\Lambda=1$, but we cannot find $\lambda$.

It seems that $M=\infty$ does not prevent the higher differentiability of the solution, actually it can increase it. For some $1<p<2$, consider the function

$$
\tilde{l}(t)= \begin{cases}\frac{1}{2}|t|^{2}+1 & \text { for }|t| \geq 1, \\ \frac{1}{p}|t|^{p}+\frac{3}{2}-\frac{1}{p} & \text { for }|t| \leq 1\end{cases}
$$

and set $\tilde{L}(\xi)=\tilde{l}(|\xi|)$. The function $\tilde{L}$ has quadratic growth.

We have, calling $H_{\tilde{L}}(\xi)$ the matrix of second derivatives of $\tilde{L}$ computed at $\xi$,

$$
H_{\tilde{L}}(\xi)= \begin{cases}(p-2)|\xi|^{p-4} \xi \otimes \xi+|\xi|^{p-2} I & \text { for }|\xi|<1 \\ I & \text { for }|\xi|>1\end{cases}
$$

so that $z^{T} H_{\tilde{L}}(\xi) z \geq|z|^{2}$ for all $\xi$, while $\left|H_{\tilde{L}}(\xi)\right| \rightarrow \infty$ as $|\xi| \rightarrow 0$.

Hence, $m=1$ while $M=\infty$. 
Let $u$ be a solution to the problem of minimizing

$$
\int_{\Omega} \tilde{L}(\nabla u(x)) d x
$$

In [3] it was proved that both $\frac{\nabla u}{|\nabla u|^{2-p}}$ and $\nabla u$ are in $W^{1,2}(\Omega)$; in particular, for every $i=1, \ldots N$, we have $\max \left\{\frac{\left|u_{x_{i}}\right|}{|\nabla u|^{2-p}},\left|u_{x_{i}}\right|\right\} \in W_{\text {loc }}^{1,2}(\Omega)$. In this case, having $M=\infty$ has increased the regularity.

A non-regular problem. Could solutions be regular, even though $L$ is not?

Consider the model problem of minimizing

$$
\int_{\Omega}[L(|\nabla u(x)|)+u(x)] d x
$$

where $L(\xi)=l(|\xi|)$, with

$$
l(t)=t+\frac{1}{2} t^{2} \quad \text { for } t \geq 0 .
$$

The convex function $t \rightarrow l(|t|)$ is continuous at 0 but it is not differentiable there; $\nabla L(\xi)=(2|\xi|+1) \frac{\xi}{|\xi|}$ is discontinuous at 0 . The corresponding EulerLagrange equation is: there exists $p(\cdot)$, a selection from $x \rightarrow \partial L(\nabla u(x))$, such that, for every $\eta$,

$$
\int_{\Omega}[\langle p(x), \nabla \eta(x)\rangle+\eta(x)] d x=0 .
$$

When $\Omega=B(0,4)$, the ball about 0 of radius 4 in the 2-dimensional Euclidean space, and $u^{0}=1$, it admits the solution

$$
u(x)= \begin{cases}0 & \text { for }|x| \leq 2, \\ \left(\frac{|x|}{2}-1\right)^{2} & \text { otherwise }\end{cases}
$$

and the function $p$ is $p(x)=\frac{1}{2} x . u$ is $C^{1}$; the second derivatives of the solution, defined for $|x| \neq 2$, have a jump discontinuity at $|x|=2$, hence the solution cannot possibly be smoother that being in $W^{2,2}(B(0,4))$.

In a recent result [6] it is shown that, indeed, a solution is in $W^{2,2}(\Omega)$. 
Violating the strict convexity condition. We said that a basic requirement for the regularity is the strict convexity condition: there exixts $m>0$ such that

$$
\langle\nabla L(x)-\nabla L(y), x-y\rangle \geq m|x-u|^{2} .
$$

Could possibly this condition be violated while we still retain the regularity of solutions?

The case $L=\frac{1}{p}|z|^{p}$. We want to see what the previous conditions and result yield, when applied to the case

$$
L=\frac{1}{p}|z|^{p} .
$$

Let us take $p>2$. Then, the condition

$$
\xi^{T} H_{L} \xi \geq \nu|\xi|^{2} \text { with } \nu>0
$$

is NOT verified. In fact, we have $\langle\nabla L(x)-\nabla L(0), x-0\rangle=|x|^{p}$ with $p>2$, contradicting the strict convexity condition near the origin.

Still, we ca find in books chapters on the regularity of solutions of problems with growth like $|\xi|^{p}$. Let us take a closer look at the conditions.

We introduce the function

$$
V(z)=\sqrt{1+|z|^{2}}
$$

since the conditions will be phrased in terms of $V$.

The conditions on $L$ are: a growth condition ( $L$ grows like $|z|^{p}$ ) and a "strict convexity" condition:

$$
\xi^{T} H_{L} \xi \geq \nu V(z)^{p-2}|\xi|^{2}
$$

with $\nu>0$. In particular, at $z=0$, the condition becomes $\xi^{T} H_{L} \xi \geq \nu|\xi|^{2}$, that is not satisfied by $|z|^{p}$ when $p>2$.

Imposing this condition removes the singularity at the origin. The modified problem has $m>0$ and $M=\infty$, but $M=\infty$ poses no problems for the regularity. In [4] the following problem was considered: for $2<p<3$, minimize

$$
\int_{\Omega}\left[\frac{1}{p}|\nabla u(x)|^{p}+g(x, u(x))\right] d x
$$

(under suitable conditions on $g$ ) and it was proved that a solution $\tilde{u}$ belongs to $W_{\text {loc }}^{2,2}(\Omega)$. Hence, the quadratic strict convexity condition is not satisfied, but the regularity of solutions still holds true. 
An idea of the proof. Fix $p=\frac{5}{2}$ so that from $l(t)=\frac{2}{5}|t|^{\frac{5}{2}}$ we obtain $l^{\prime \prime}(t)=\frac{3}{2}|t|^{\frac{1}{2}}$ so that as $|t| \rightarrow 0$, we have $l^{\prime \prime}(t) \rightarrow 0$ and the strict convexity condition is violated.

Consider the one-dimensional problem of minimizing

$$
\int_{\alpha}^{\beta}\left[\frac{2}{5}\left|x^{\prime}(t)\right|^{\frac{5}{2}}+f(t) x(t)\right] d t, \quad y(\alpha)=a, \quad y(\beta)=b .
$$

If we insert in the Euler-Lagrange equation the standard variation $\phi$ defined by $\phi(t)=\eta^{2}(t) x^{\prime}(t)$, we obtain

$$
\int_{\alpha}^{\beta} \frac{3}{2}\left|x^{\prime}\right|^{\frac{1}{2}}\left(x^{\prime \prime}(t)\right)^{2} \eta^{2}=-\int_{\alpha}^{\beta} \frac{3}{2}\left|x^{\prime}\right|^{\frac{1}{2}}\left(x^{\prime \prime}(t) x^{\prime} 2 \eta \eta^{\prime}+\ldots\right.
$$

( $\ldots$ represent the terms arising from $g(x, u)$ that we do not wish to discuss here).

Since $\left|x^{\prime}\right|^{\frac{1}{2}} \rightarrow 0$ for $\left|x^{\prime}\right|$ small, we cannot obtain a positive lower bound for

$$
\int_{\alpha}^{\beta}\left(x^{\prime \prime}(t)\right)^{2} .
$$

Hence, the idea of the proof is to contrast the $\infty$ with a 0 .

Assume that as a variation $\phi$ we take instead $\phi(t)=\eta^{2}(t) \gamma\left(x^{\prime}(t)\right)$ and we choose

$$
\gamma(t)=|t|^{\frac{1}{2}} \operatorname{sign}(t)
$$

so that $\gamma^{\prime}(t)=\frac{1}{2} \frac{1}{|t|^{\frac{1}{2}}}$; then, the Euler-Lagrange equation gives

$$
\int_{\alpha}^{\beta} \frac{3}{2}\left|x^{\prime}\right|^{\frac{1}{2}} \frac{1}{2} \frac{1}{\left|x^{\prime}\right|^{\frac{1}{2}}}\left(x^{\prime \prime}(t)\right)^{2} \eta^{2}=-\int_{\alpha}^{\beta} \frac{3}{2}\left|x^{\prime}\right|^{\frac{1}{2}}\left(x^{\prime \prime}(t)\left|x^{\prime}\right|^{\frac{1}{2}} \operatorname{sign}\left(x^{\prime}\right) 2 \eta \eta^{\prime}+\ldots\right.
$$

and we can obtain a bound for $\int_{\alpha}^{\beta}\left(x^{\prime \prime}(t)\right)^{2}$.

The idea works for $2<p<3$. It stops working at $p=3$ : in fact, $p=3$ would give that $l^{\prime \prime}\left(x^{\prime}\right)=x^{\prime}$; to contrast it we would need $\gamma^{\prime}(t)=\frac{1}{t}$ whose integral is $\ln (t)$, unbounded near the origin.

We have shown that there exists at least one case where the functional to be minimized needs not satisfy the quadratic strict convexity condition. Could this condition be weaken in general? 


\section{A different regularity problem}

When the integration set is one-dimensional, it follows that the dual variable $p(t)=\nabla L\left(x^{\prime}(t)\right)$ is absolutely continuous, even when $L$ is not differentiable and we consider a selection $p(\cdot)$ from $\partial L(\nabla u(\cdot))$. Can we hope to prove the regularity of a selection $p(x) \in \partial L(\nabla u(x))$ ?

An example. We consider the problem of minimizing

$$
I(v)=\int_{\Omega}[L(\nabla v(x))+v(x)] d x
$$

on $u^{0}+W_{0}^{1,2}(\Omega)$, where

$$
L(\xi)=l(|\xi|)=\frac{1}{2}\left(|\xi|^{2}-1\right)^{+}
$$

and $\Omega \subset \mathbb{R}^{2}$. Since

$$
l^{\prime}(t)= \begin{cases}0 & \text { for }|t| \leq 1 \\ t & \text { for }|t|>1\end{cases}
$$

the gradient of $L$ is discontinuous when $|\xi|=1$; hence, the function $L$ presents at once a flat part $(|\xi| \leq 1$, where $L \equiv 0)$ and a discontinuity of the gradient.

A (radial) solution $u$ to the problem of minimizing (4.4) is given by

$$
u(x)= \begin{cases}|x| & \text { for }|x| \leq 2 \\ \frac{1}{4}|x|^{2}+1 & \text { for }|x| \geq 2\end{cases}
$$

whose gradient is

$$
\nabla u(x)= \begin{cases}\frac{x}{|x|} & \text { for }|x|<2 \\ \frac{1}{2} x & \text { for }|x|>2\end{cases}
$$

so that the gradient of $u$ is discontinuous at $x=0$.

From the known results on the validity of the Euler-Lagrange equation [11, we infer the existence of a $p \in L^{2}(\Omega)$ such that, at once, $p$ is a selection from $x \rightarrow \partial L(\nabla u(x))$ and such that $\operatorname{div}(p)=1$; when $|x|>2$ we have $|\nabla u(x)|>1$ and $p(x)=\nabla L(\nabla u(x))=\nabla u(x)=\frac{1}{2} x$; then, we notice that the very same function $\frac{1}{2} x$ is, even for $|x| \leq 2$, a selection from $\partial L(\nabla u(x))$, has (weak) divergence $\equiv 1$ and is everywhere differentiable, even at the origin 0 , where $\nabla u$ is discontinuous. On this example, $p$ is more regular than $\nabla u$.

Hence, from the example, it seems that the regularity of $\partial L(\nabla u)$ might hold under broader conditions than the regularity of $\nabla u$. 
Can this fact be shown for general (not rotational) boundary conditions? The author has found the problem of proving the regularity of $p$ to be particularly difficult.

\section{References}

[1] Brézis H., Multiplicateur de Lagrange en Torsion Elastoplastique, Arch. Rat. Mech. Anal. 49 (1972), 32-40.

[2] Cellina A., The classical problem of the calculus of variations in the autonomous case: relaxation and Lipschitzianity of solutions, Trans. Amer. Math. Soc. 356 (2004), 415426.

[3] Cellina A., Strict convexity and the regularity of solutions to variational problems, ESAIM Control Optim. Calc. Var. 22 (2016), 862-871.

[4] Cellina A., The regularity of solutions to the $p$-Laplace equation for $1<p<2$, ESAIM Control Optim. Calc. Var. To appear.

[5] Cellina A., The validity of the Euler-Lagrange equation for solutions to variational problems, J. Fixed Point Theory Appl. 15 (2014), 577-586.

[6] Cellina A., A case of regularity of solutions to nonregular problems, SIAM J. Control Optim. 53 (2015), 2835-2845.

[7] Cellina A., Ferriero A., Existence of Lipschitzian solutions to the classical problem of the calculus of variations in the autonomous case, Ann. Inst. H. Poincaré Anal. Non Linéaire 20 (2003), 911-919.

[8] Cellina A., Perrotta S., On minima of radially symmetric functionals of the gradient, Nonlinear Anal. 23 (1994), no. 2, 239-249.

[9] Cellina A., Staicu V., The existence of solutions to variational problems of slow growth, J. Differential Equations 260 (2016), 5834-5846.

[10] Cellina A., Treu G., Zagatti S., On the minimum problem for a class of non-coercive functionals, J. Differential Equations 127 (1996), 225-262.

[11] Dacorogna B., Direct methods in the Calculus of Variations, Second edition, Springer, Berlin, 2008.

[12] Degiovanni M., Marzocchi M., On the Euler-Lagrange equation for functionals of the calculus of variations without upper growth conditions, SIAM J. Control Optim. 48 (2009), 2857-2870.

[13] Esposito L., Leonetti F., Mingione G., Regularity results for minimizers of irregular integrals with ( $p, q)$ growth, Forum Math. 14 (2002), 245-272.

[14] Jenkins H., Serrin J., The Dirichlet problem for the minimal surface equation in higher dimensions, J. Reine Angew. Math. 229 (1968), 170-187.

[15] Rockafellar R.T., Convex Analysis, Princeton University Press, Princeton, 1972.

Dipartimento di Matematica e Applicazioni

Università degli Studi di Milano-Bicocca

Via R. Cozzi 53

I-20125 Milano

ITALY

e-mail: arrigo.cellina@unimib.it 